\title{
Dynamic Load Sharing Behaviours of Planetary Gear Trains and Parameter Study through Perturbation Analysis
}

\author{
Chao Xun $\mathbb{D D}^{1}$ and He Dai ${ }^{2}$ \\ ${ }^{1}$ School of Mechanical Engineering, Nanjing Institute of Technology, Nanjing 211167, China \\ ${ }^{2}$ State Key Laboratory of Mechanical System and Vibration, School of Mechanical Engineering, Shanghai Jiao Tong University, \\ 800 Dongchuan Rd, Shanghai 200240, China \\ Correspondence should be addressed to Chao Xun; xunchao0130@126.com
}

Received 24 September 2020; Revised 3 March 2021; Accepted 22 March 2021; Published 12 April 2021

Academic Editor: Zengshun Chen

Copyright (C) 2021 Chao Xun and He Dai. This is an open access article distributed under the Creative Commons Attribution License, which permits unrestricted use, distribution, and reproduction in any medium, provided the original work is properly cited.

\begin{abstract}
In this paper, an analytical solution on the dynamic mesh forces of planetary gear trains (PGTs) is proposed by investigating a lumped-parameter model. By using the method of multiple-scales (MMS), closed-form expressions of mesh force under the effects of manufacturing and assembly errors are obtained. From these expressions, the effects of several key factors such as the tooth thickness error, pin position error, applied torque, support stiffness of sun gear, and tooth profile modifications (TPM) on dynamic load sharing behaviours are explored. Numerical integration is carried out to verify the validation of the proposed method, and the developed expressions are also validated by comparing the results with previously published predictions. The results for several examined PGT systems show that the key factors abovementioned affect the dynamic load sharing behaviours as both static and dynamic factors. An important new conclusion obtained by this work is that proper tooth profile modifications keep the dynamic load sharing factors almost equal to the results obtained under static conditions. This conclusion provides the possibility to simplify the dynamic analysis to the static analysis on the dynamic load sharing problems.
\end{abstract}

\section{Introduction}

In the applications of high-power mechanical transmission areas, to guarantee the load on each component within the safe ranges, split-torque transmission systems are widely used. Planetary gear train (PGT) is one of the most popular split-torque transmission systems, because of its advantages such as high-power density, high transmission ratio, low bearing load, and compactness. Theoretically, the input torque should be shared evenly by all the planets. In practical applications, however, each path of a PGT will carry uneven load due to the presence of errors, such as tooth thickness error and pin position error. The load sharing behaviours are affected by a variety of factors such as the gravity, support stiffness of central components, bearing clearance, backlashes, applied torque, and component flexibility [1]. Uneven load sharing leads one or several planet gears to carry load which is more than the nominal value, which shortens the lifetime of PGT systems and increases potential damaging risk.
In the past 20 years, nonlinearities in geared systems caused by factors including backlash [2], time-varying mesh stiffness [3], friction [4] between the mating gear teeth, and the error of the gear transmission have been the main concerns of researchers. Neglecting the effects of dynamic vibrations, the quasistatic load sharing behaviours of PGTs have been extensively studied [5]. Previous quasistatic analysis showed that the load sharing behaviours of PGTs are sensitive to the manufacturing errors [6], and this sensitivity increases with the increasing of the number of planet gears [7]. The results obtained from finite element models showed that load sharing is dramatically affected by the applied torque [8]. Floating one or several central components can improve the load sharing and decrease critical tooth stress [9]. These aforementioned conclusions obtained by quasistatic analysis have been confirmed by experimental results [10-12]. Ligata et al. [13] presented planet load sharing formulas under quasistatic conditions, and these formulas revealed the influence of various parameters and errors on 
the load sharing. Singh $[14,15]$ gave a physical explanation for the basic mechanism which causes the unequal load sharing phenomenon. Quasistatic analysis shows the mechanism of the influence of various parameters and errors on the load sharing as static factors and provides researchers a basic understanding on the loading sharing problems. However, these studies cannot explore the effects of the dynamic factors which are unavoidable in practical PGTs under the actual working conditions.

In order to further predict the load sharing behaviours under practical dynamic working conditions, some researchers investigated the dynamic load sharing by using lumped-parameter models. Kahraman [16] established a relationship between dynamic load and static load by defining the dynamic load factor to indicate the effects of dynamic factors on mesh load. Considering the planet pin position errors [17, 18] and eccentricities [19], Gu and Velex investigated the influence of centrifugal forces and pin flexibility on system with rotating carriers. Results indicate the centrifugal forces of rotating carrier may change the dynamic load sharing behaviours. It is believed that the manufacturing errors may significantly increase the dynamic vibrations [20-22], and the dynamic behaviours have different sensitivity to different kinds of errors [23]. Mo et al. $[24,25]$ conducted analytical investigation of load sharing characteristics for herringbone planetary gear train with flexible support and multipower face gear split flow system. The impacts of manufacturing errors, assembly errors, manufacturing error phases, assembly error phases, meshing damping, support stiffness, and the input power on the load sharing coefficients were analyzed. The load sharing may be improved by modifying the tooth profile [26] and floating one central component [27]. However, the floating central component will lead large vibrations [28]. The aforementioned dynamic analysis was proposed by numerical methods. These numerical methods may provide accurate solutions; however, they were not able to reveal the mechanism of the influence of the system parameters and the variety of errors on the dynamic load sharing.

In this effort, an analytical solution on the dynamic load sharing behaviours of PGTs is proposed by investigating a lumped-parameter model. Based on the previous works $[29,30]$ and the authors' efforts [31, 32], the method of multiple-scales (MMS) is applied to analyse the dynamic load sharing behaviours under the effects of tooth thickness errors and the pin position errors for the first time. Closedform expressions of mesh force versus mesh frequency are derived by MMS. Through these expressions, the effects of several key parameters, tooth thickness errors, pin position errors, and tooth profile modifications (TPM) on the dynamic load sharing behaviours are illustrated. Numerical integration is employed to verify the proposed method. This study provides guidance for the selections of the key parameters of the PGT systems. The rest of this article is organized as follows. In the second section, the modelling and problem definition are presented. In the third section, closed-form expressions of mesh forces are driven through the method of multiple-scales (MMS). Analytical and numerical results and discussions are presented in the fourth section. Finally, conclusions are collected together and presented.

\section{Model and Problem Definition}

2.1. Modelling with Various Kinds of Errors. A lumped-parameter model of PGTs with a sun gear, a fixed carrier, a ring gear, and $N$ planet gears is shown in Figure 1. In this model, only the rotational motions of each component and two translational motions of the sun gear are considered. The number of degree of freedom is $N+5$. In Figure $1, k_{b s}$ is the translational stiffness of the sun gear. $k_{s p}$ and $k_{r p}$ are the mesh stiffness of the pth s-p and r-p mesh, respectively. $k_{s u}, k_{r u}$, and $k_{c u}$ are the torsional stiffness of sun, ring, and carrier, respectively. $u$ denotes the rotational motions.

Figures 2 and 3 are two diagrams to describe the r-p and s-p mesh, respectively. In these two figures, $e$ and $h$ are equivalent tooth thickness errors and the gap induced by tooth profile modifications, respectively. $\widehat{\delta}_{r p}$ and $\widehat{\delta}_{s p}$ are respectively the relative mesh deformation on r-p and s-p meshes caused by the motions of the components. Considering the mesh force between the ring gear and planet gears, one can write the equation of ring gear as

$$
\begin{gathered}
\frac{J_{r}}{r_{r}^{2}} \ddot{u}_{r}+c_{u r} \dot{u}_{r}+k_{r u} u_{r}+\sum_{p=1}^{N} k_{r p}(t) \Theta\left(\delta_{r p}\right) \widehat{\delta}_{r p} \\
-\sum_{p=1}^{N} \widehat{f}_{e r p}+\sum_{p=1}^{N} \widehat{f}_{m r p}=0,
\end{gathered}
$$

where

$$
\begin{aligned}
& \widehat{\delta}_{r p}=-u_{c} \cos \alpha_{r}+u_{r}-u_{p}, \\
& \delta_{r p}=-u_{c} \cos \alpha_{r}+u_{r}-u_{p}-e_{r p}+h_{r p},
\end{aligned}
$$

where $\hat{f}_{e r p}$ and $\hat{f}_{m r p}$ are respectively the additional mesh forces induced by errors and TMP. $c$ denotes the damping term, and $\alpha_{r}$ is the pressure angle of r-p mesh. $J$ is the moment and $r$ is the base radius. $e_{r p}$ and $h_{r p}$ are respectively the tooth thickness errors and TPM functions on r-p mesh.

The tooth separation is modelled by $\Theta(\delta)$, where $\delta$ is the deformation of the gear mesh. Figure 4 shows the effects of the backlash on the mesh force of the tooth pair. When $\delta>0$, where $\delta$ is the deformation of the gear mesh, the tooth pair maintains contact; when $-b<\delta<0$, where $b$ is the width of the backlash, contact loss occurs, and when $\delta<-b$, backside tooth contact occurs. Backside tooth contact is not normally observed in practice because of the gear preload and backlash, so it is neglected. The tooth separation functions $\Theta(\delta)$ are

$$
\Theta(\delta)= \begin{cases}1, & \delta \geq 0 \\ 0, & \delta<0 .\end{cases}
$$

The sun gear links with $N$ planet gears and the equation of motion could be expressed as 


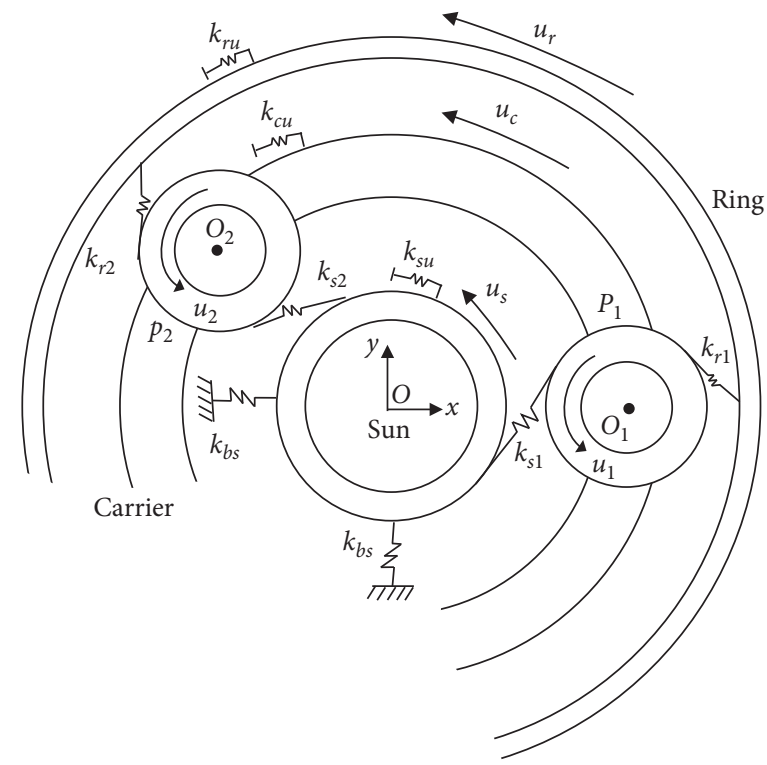

Figure 1: Lumped-parameter model of PGTs.

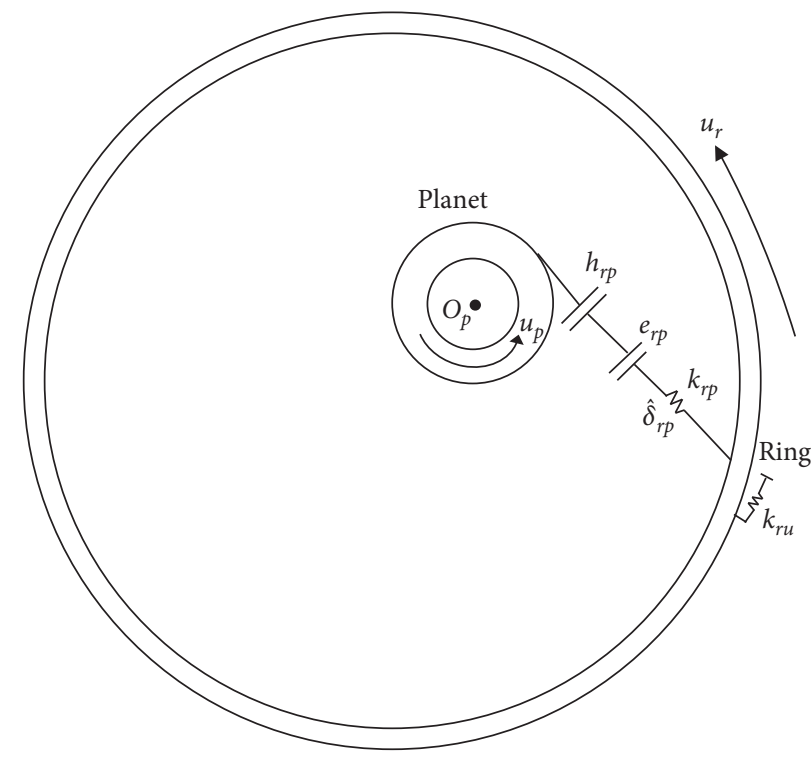

FIgURE 2: Diagram of r-p mesh.

$$
\begin{aligned}
& m_{s} \ddot{x}_{s}+c_{x s} \dot{x}_{s}+k_{b s} x_{s}+\sum_{p=1}^{N} k_{s p}(t) \Theta\left(\delta_{s p}\right) \widehat{\delta}_{s p}\left[-\sin \left(\psi_{s p}\right)\right]-\sum_{p=1}^{N} \widehat{f}_{e x s p}+\sum_{p=1}^{N} \widehat{f}_{m x s p}=0 \\
& m_{s} \ddot{y}_{s}+c_{y s} \dot{y}_{s}+k_{b s} y_{s}+\sum_{p=1}^{N} k_{s p}(t) \Theta\left(\delta_{s p}\right) \widehat{\delta}_{s p} \cos \left(\psi_{s p}\right)-\sum_{p=1}^{N} \widehat{f}_{e y s p}+\sum_{p=1}^{N} \widehat{f}_{m y s p}=0, \\
& \frac{J_{s}}{r_{s}^{2}} \ddot{u}_{s}+c_{u s} \dot{u}_{s}+k_{s u} u_{s}+\sum_{p=1}^{N} k_{s p}(t) \Theta\left(\delta_{s p}\right) \widehat{\delta}_{s p}-\sum_{p=1}^{N} \widehat{f}_{\text {eusp }}+\sum_{p=1}^{N} \widehat{f}_{\text {musp }}=\frac{T_{s}}{r_{s}},
\end{aligned}
$$




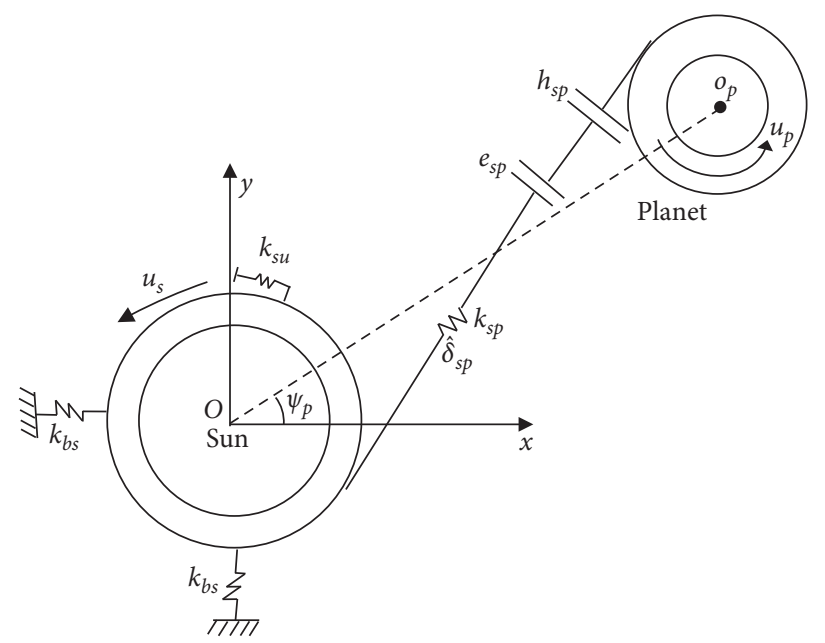

Figure 3: Diagram of s-p mesh.

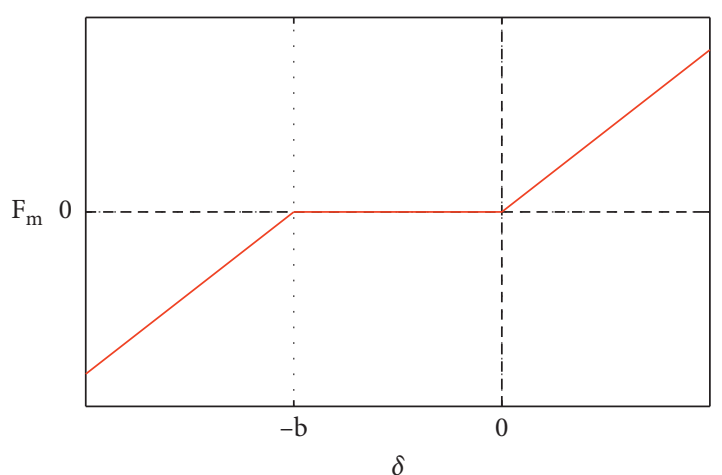

Figure 4: Backlash and tooth separations.

where

$$
\begin{aligned}
\widehat{\delta}_{s p}= & -u_{c} \cos \alpha_{s}-x_{s} \sin \psi_{s p}+y_{s} \cos \psi_{s p}+u_{s}+u_{p}, \\
\delta_{s p}= & -u_{c} \cos \alpha_{s}-x_{s} \sin \psi_{s p}+y_{s} \cos \psi_{s p}+u_{s} \\
& +u_{p}-e_{s p}+h_{s p},
\end{aligned}
$$

where $x_{s}$ and $y_{s}$ are the translational motions of sun gear in the $x$ and $y$ directions, respectively, $\alpha_{s}$ is the pressure angle of s-p mesh, $\Psi_{p}$ is the angle between $x$-axis and the line oo ${ }_{p}$ and $\Psi_{s p}=\Psi_{p}-\alpha, T_{s}$ is the transmitted torque applying on sun gear, and $e_{s p}$ and $h_{s p}$ are respectively the tooth thickness errors and TPM functions on s-p mesh. From equations (1) and (4), the equation of motion associated with the $p$ th planet gear is

$$
\begin{aligned}
& \frac{J_{p}}{r_{p}^{2}} \ddot{u}_{p}+c_{u p} \dot{u}_{p}+k_{s p}(t) \Theta\left(\delta_{s p}\right) \widehat{\delta}_{s p}-k_{r p}(t) \Theta\left(\delta_{r p}\right) \widehat{\delta}_{r p} \\
& -\widehat{f}_{e s p}+\widehat{f}_{e r p}+\widehat{f}_{m s p}-\widehat{f}_{m r p}=0 .
\end{aligned}
$$

As shown in Figure 5, all the planet gears are fixed on the carrier and the equation of carrier rotational motion is

$$
\begin{gathered}
\left(\frac{J_{c}}{r_{c}^{2}}+N m_{p}\right) \ddot{u}_{c}+c_{u c} \dot{u}_{c}+k_{c u} u_{c}-\sum_{p=1}^{N} k_{s p}(t) \Theta\left(\delta_{s p}\right) \widehat{\delta}_{s p} \cos \left(\alpha_{s}\right)-\sum_{p=1}^{N} k_{r p}(t) \Theta\left(\delta_{r p}\right) \widehat{\delta}_{r p} \cos \left(\alpha_{r}\right) \\
+\sum_{p=1}^{N} \widehat{f}_{e s p} \cos \left(\alpha_{s}\right)+\sum_{p=1}^{N} \widehat{f}_{e r p} \cos \left(\alpha_{r}\right)-\sum_{p=1}^{N} \widehat{f}_{m s p} \cos \left(\alpha_{s}\right)-\sum_{p=1}^{N} \widehat{f}_{m r p} \cos \left(\alpha_{r}\right)=0,
\end{gathered}
$$

where $r_{c}$ is the length of oo $_{1}$, as shown in Figure 1.

As shown in Figure 6(a), the tooth thickness error is the deviation between the actual tooth thickness and the design involute tooth thickness. When the actual tooth is thicker, the additional mesh force is positive and vice versa. The pin position error is shown in Figure 6(b). In a rotating component, both time-varying and time-invariant pin position errors exist. The time-varying pin position errors are induced in the manufacturing process. The direction of these manufacturing pin position errors changes with the rotating of the component. The time-invariant pin position errors are induced in the assemble process. The values and the directions of the assemble pin position errors are consistent once the component assembled.

Figure 7 shows how different errors affect the equivalent tooth thickness errors. For the sake of simplicity, the carrier 


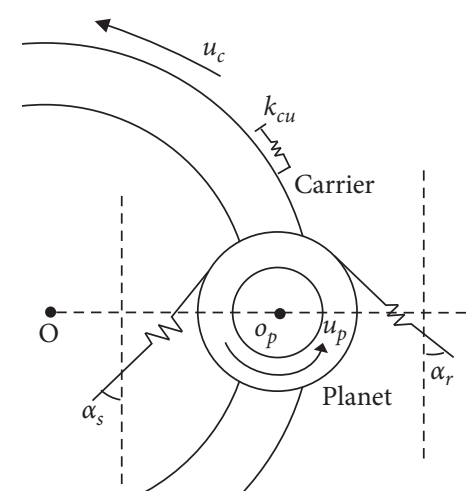

FIGURE 5: Diagram of carrier with planet gear.

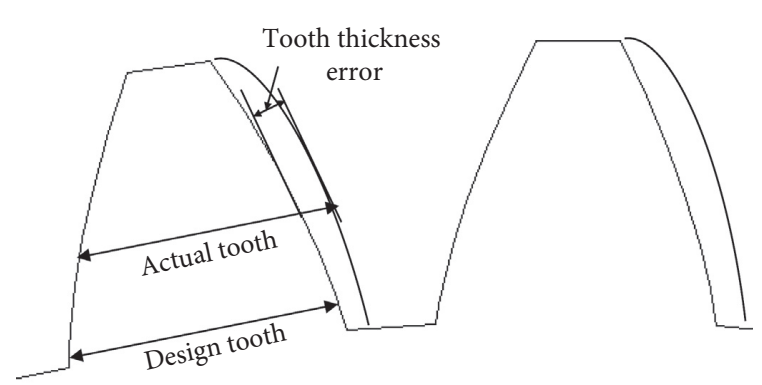

(a)

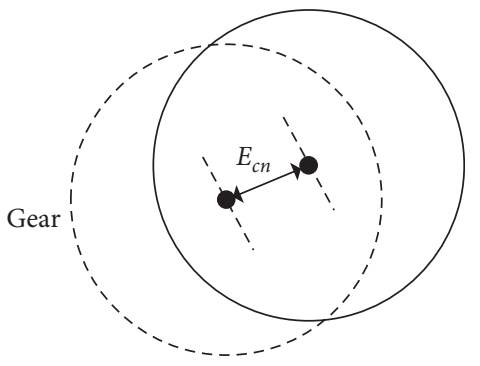

(b)

FIgURE 6: Tooth thickness error and pin position error.

is not shown. Converting all the errors to the gear pair meshing lines, the equivalent tooth thickness errors obtained through superposition could be expressed as

$$
\begin{aligned}
e_{s p}= & \bar{E}_{p s}+\bar{E}_{p p}+\bar{E}_{c c} \cos \left(\varphi_{s p}^{c}\right)+\bar{E}_{c s} \cos \left(\varphi_{s p}^{s}\right)+\bar{E}_{c p} \cos \left(\varphi_{p p}^{s}\right)+\widetilde{E}_{s p} \cdot \sin \left(\omega_{m} t+\gamma_{s p}\right) \\
& +\widetilde{E}_{c s} \cdot \sin \left[\left(\Omega_{s}-\Omega_{c}\right) \cdot t+\gamma_{s p}^{s}\right]+\widetilde{E}_{c p} \cdot \sin \left[\left(\Omega_{p}-\Omega_{c}\right) \cdot t+\gamma_{p p}^{s}\right], \\
e_{r p}= & \bar{E}_{p r}+\bar{E}_{p p}+\bar{E}_{c c} \cos \left(\varphi_{r p}^{c}\right)+\bar{E}_{c r} \cos \left(\varphi_{r p}^{r}\right)+\bar{E}_{c p} \cos \left(\varphi_{p p}^{r}\right)+\widetilde{E}_{r p} \cdot \sin \left(\omega_{m} t+\gamma_{r p}\right) \\
& +\widetilde{E}_{c r} \cdot \sin \left[\left(\Omega_{r}-\Omega_{c}\right) \cdot t+\gamma_{r p}^{r}\right]+\widetilde{E}_{c p} \cdot \sin \left[\left(\Omega_{p}-\Omega_{c}\right) \cdot t+\gamma_{p p}^{r}\right] .
\end{aligned}
$$

In equation (8), $E_{p r}, E_{p s}$, and $E_{p p}$ represent the tooth thickness errors of ring, sun gear, and the pth planet gear, respectively. $E_{c c}, E_{c r}, E_{c s}$, and $E_{c p}$ represent the pin position errors of the carrier, ring, sun gear, and the $p$ th planet gear, respectively. $\bar{E}$ is the time-invariant component and $\widetilde{E}$ is the amplitude of the time-varying component. $\widetilde{E}_{s p}$ and $\widetilde{E}_{r p}$ are the amplitudes of the tooth thickness errors of s-p and r-p mesh, respectively. $\varphi$ is the angle between the directions of the corresponding pin position error and the mesh line, and $\gamma$ is the initial phase of the time-varying errors. $\Omega$ is the rotational speed and $t$ denotes time. $\omega_{m}$ is the mesh frequency, which is determined by the gear tooth and the power flow path as a function of rotational speed, as given in the following equation [16]:

$$
\omega_{m}= \begin{cases}\frac{Z_{s} Z_{r} \Omega_{s}}{\left(Z_{s}+Z_{r}\right)}, & \text { fixed ring gear, } \\ \frac{Z_{s} Z_{r} \Omega_{r}}{\left(Z_{s}+Z_{r}\right)}, & \text { fixed sun gear, } \\ Z_{s} \Omega_{s}\left(\text { or } Z_{r} \Omega_{r}\right), & \text { fixed carrier, }\end{cases}
$$




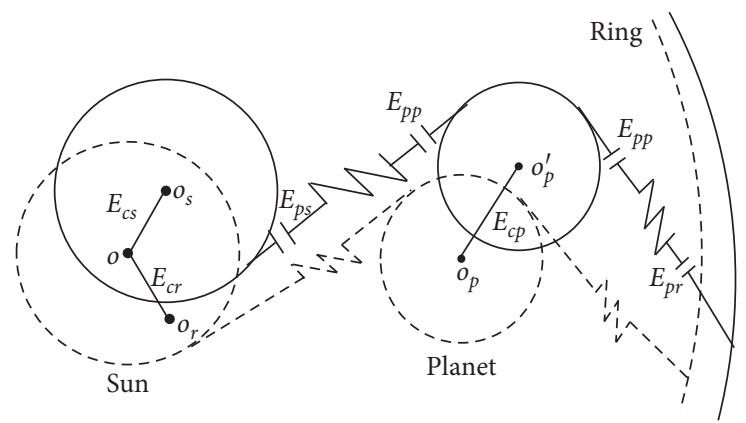

Figure 7: Contributions of different errors to the equivalent tooth thickness errors.

where $Z_{s}$ and $Z_{r}$ are the tooth number of sun gear and ring gear, respectively.

Then, the additional mesh force introduced by equivalent tooth thickness errors on the $p$ th s-p mesh and r-p mesh is obtained,

$$
\begin{aligned}
& \hat{f}_{e s p}(\mathbf{x}, t)=k_{s p}(t) \Theta\left(\delta_{s p}\right) e_{s p}, \\
& \hat{f}_{\text {erp }}(\mathbf{x}, t)=k_{r p}(t) \Theta\left(\delta_{r p}\right) e_{r p} .
\end{aligned}
$$

There are several methods to modify gear tooth surfaces, including crowning, tip relief, and root relief with linear or parabolic variations with roll angle. The TPM curves, the magnitude of the relief, and the modification length are the three key factors that determine the effects of the TPM on vibration reduction. Without loss of generality, linear relief is applied to double tooth pair contact areas about the tooth tip and root in this study. As shown in Figure 8, for the spur PGTs, TPM is applied on the double teeth contact area about the tooth tip and root. The additional mesh force introduced by TPM on the $p$ th s-p and r-p mesh could be expressed as

$$
\begin{aligned}
& \widehat{f}_{m s p}(\mathbf{x}, t)=k_{s p}(t) \Theta\left(\delta_{s p}\right) h_{s p}, \\
& \widehat{f}_{m r p}(\mathbf{x}, t)=k_{r p}(t) \Theta\left(\delta_{r p}\right) h_{r p} .
\end{aligned}
$$

From equations (1)-(7), the system equation in matrix form is

$$
\begin{aligned}
& \mathbf{M} \ddot{\mathbf{x}}+\mathbf{C} \dot{\mathbf{x}}+\left[\mathbf{K}_{b}+\mathbf{K}_{m 0}+\mathbf{K}_{m v}(\mathbf{x}, t)\right] \mathbf{x}-\mathbf{F}_{d}(\mathbf{x}, t)+\mathbf{F}_{m}(\mathbf{x}, t)=\mathbf{F}_{t}, \\
& \mathbf{x}=\left[u_{c}, u_{r}, x_{s}, y_{s}, u_{s}, u_{1}, \ldots, u_{N}\right]^{T} \text {. }
\end{aligned}
$$

$\mathbf{M}$ is the mass matrix,

$$
\mathbf{M}=\operatorname{diag}\left[\frac{J_{c}}{r_{c}^{2}}+N m_{p}, \frac{J_{r}}{r_{r}^{2}}, m_{s}, m_{s}, \frac{J_{s}}{r_{s}^{2}}, \frac{J_{1}}{r_{1}^{2}}, \ldots, \frac{J_{N}}{r_{N}^{2}}\right] .
$$

$\mathbf{K}_{b}$ is the support stiffness matrix between the PGT and the fixture. $\mathbf{K}_{m v}$ and $\mathbf{K}_{m 0}$ are the varying part and mean part of stiffness matrix, respectively. 


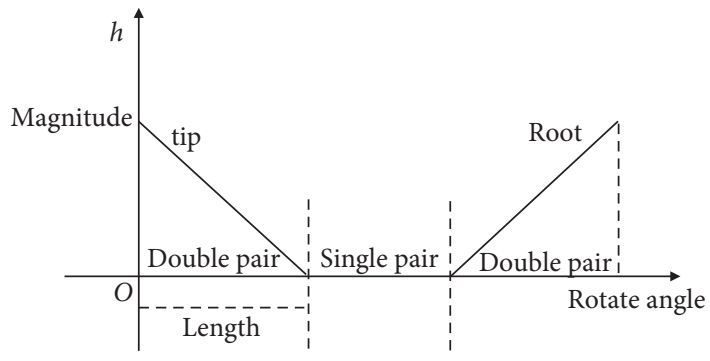

Figure 8: Tooth profile modification.

$$
\begin{aligned}
\mathbf{K}_{b} & =\operatorname{diag}\left[k_{c u}, k_{r u}, k_{b s}, k_{b s}, k_{s u}, 0, \ldots, 0\right], \\
\mathbf{K}_{m 0} & =\sum_{p=1}^{N}\left(\bar{k}_{s p} \mathbf{K}_{s p}+\bar{k}_{r p} \mathbf{K}_{r p}\right), \\
\mathbf{K}_{m v}(\mathbf{x}, t) & =\sum_{p=1}^{N}\left[\widetilde{k}_{s p}(\mathbf{x}, t) \mathbf{K}_{s p}+\widetilde{k}_{r p}(\mathbf{x}, t) \mathbf{K}_{r p}\right],
\end{aligned}
$$

where $\bar{k}$ and $\widetilde{k}$ are the average and varying components of the time-varying mesh stiffness, respectively. Considering the tooth separation, one can write the mesh stiffness as

$$
\begin{aligned}
& k_{s p}(\mathbf{x}, t)=k_{s p}(t) \Theta\left(\delta_{s p}\right)= \begin{cases}k_{s p}(t), & \delta_{s p} \geq 0, \\
0, & \delta_{s p}<0,\end{cases} \\
& k_{r p}(\mathbf{x}, t)=k_{r p}(t) \Theta\left(\delta_{r p}\right)= \begin{cases}k_{r p}(t), & \delta_{r p} \geq 0, \\
0, & \delta_{r p}<0 .\end{cases}
\end{aligned}
$$

In this study, the rectangle waves [30,33] are applied to approximate the time-varying mesh stiffness. As shown in Figure 9, mesh stiffness varies as the number of contact tooth pair changes. $\mathbf{K}_{s p}$ is the nondimensional mesh stiffness matrix and can be written as

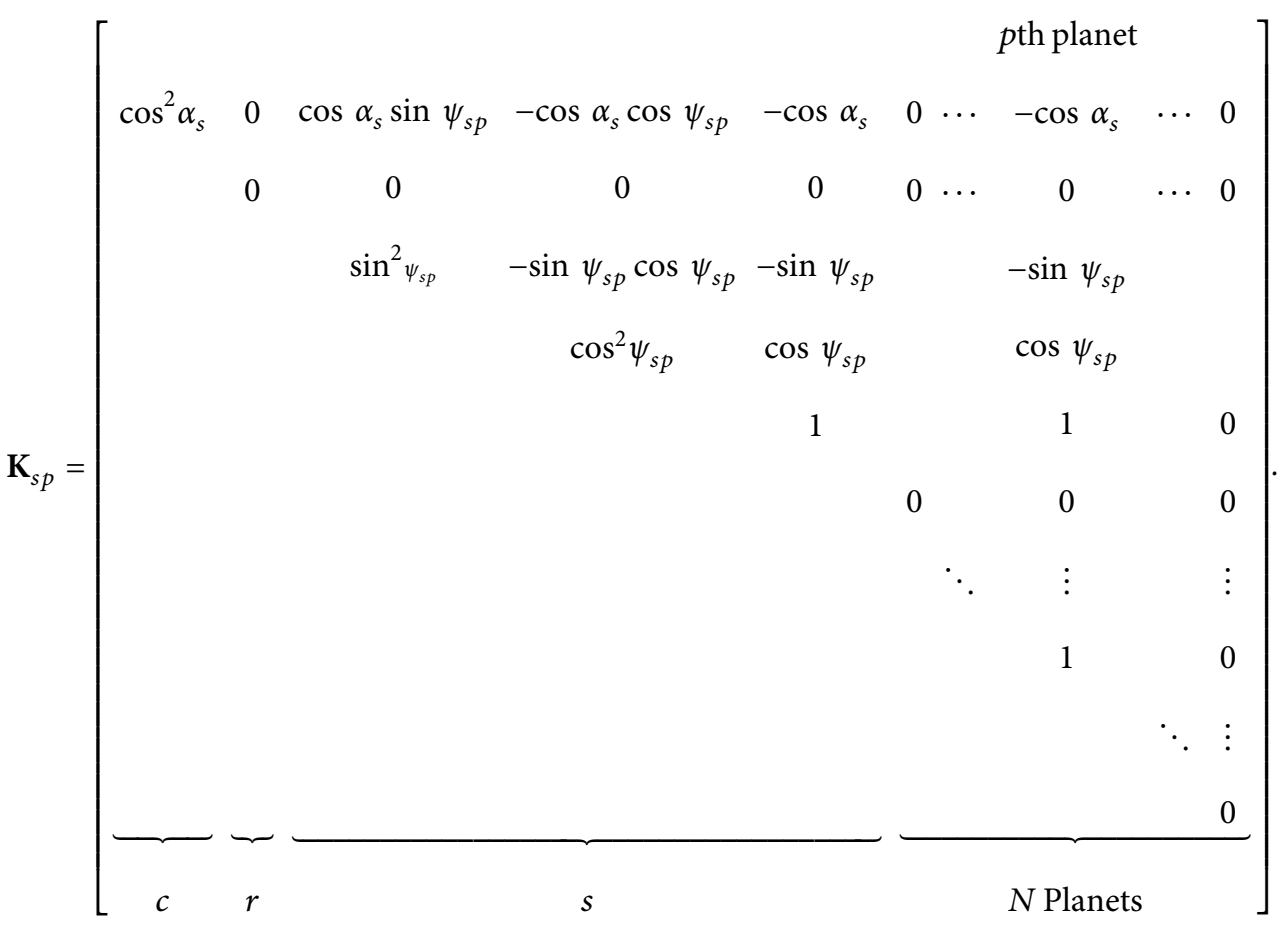

$\mathbf{K}_{r p}$ has a similar form as $\mathbf{K}_{s p}$.

$\mathrm{C}$ is the damping matrix in the form of

$$
\mathbf{C}=\alpha \mathbf{M}+\beta\left(\mathbf{K}_{b}+\mathbf{K}_{m 0}\right) .
$$

$\mathbf{F}_{t}$ is the external load vector. $\mathbf{F}_{d}$ and $\mathbf{F}_{m}$ are respectively the inner force vectors introduced by the equivalent tooth thickness errors and TPM. 


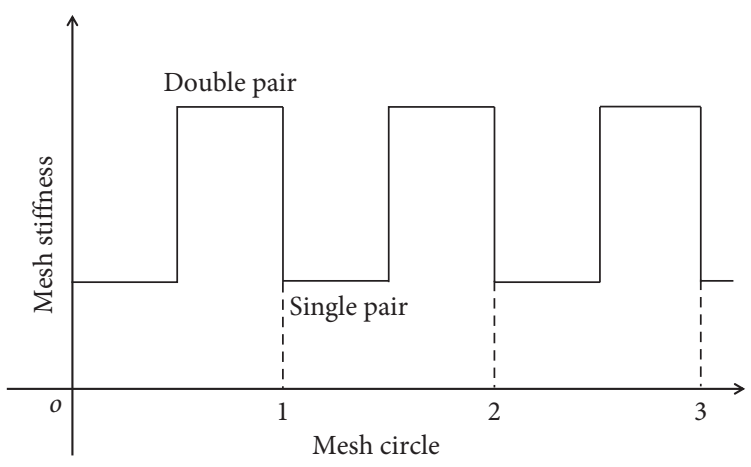

FIgure 9: Time-varying mesh stiffness.

$$
\begin{aligned}
& \mathbf{F}_{d}(\mathbf{x}, t)=\sum_{p=1}^{N}\left[\hat{f}_{\text {esp }}(\mathbf{x}, t) \mathbf{D}_{s p}+\hat{f}_{e r p}(\mathbf{x}, t) \mathbf{D}_{r p}\right] \\
& \mathbf{F}_{m}(\mathbf{x}, t)=\sum_{p=1}^{N}\left[\hat{f}_{m s p}(\mathbf{x}, t) \mathbf{D}_{s p}+\hat{f}_{m r p}(\mathbf{x}, t) \mathbf{D}_{r p}\right], \\
& \mathbf{D}_{s p}=[\underbrace{-\cos \alpha_{s}, 0,-\sin \psi_{s p}, \cos \psi_{s p} 1,}_{c, r, s} \underbrace{0, \cdots,}_{N \text { Planets }} \begin{array}{c}
\text { pth planet } \\
0, \quad \cdots, 0
\end{array}]^{T}, \\
& \mathbf{D}_{r p}=[\underbrace{-\cos \alpha_{r}, 1,0,0,0,}_{c, r, s} \underbrace{0, \cdots, c^{2}, 1, \quad \cdots, 0}_{\text {N Planets }}]^{T} \text {, }
\end{aligned}
$$

where $\mathbf{D}_{s p}$ and $\mathbf{D}_{r p}$ are the component vectors of $\hat{f}_{\text {es } p}(\mathbf{x}, t)$ and $\widehat{f}_{\text {erp }}(\mathbf{x}, t)$ on each degree of freedom. The time-varying mesh stiffnesses and the additional mesh forces induced by errors and TPM can be expressed in terms of Fourier series as

$$
\begin{gathered}
k_{s p}(t)=\bar{k}_{s p}+\left(\sum_{l=1}^{\infty} \tilde{k}_{s p}^{(l)} e^{j l \omega_{m} t}+c . c .\right), \\
k_{r p}(t)=\bar{k}_{r p}+\left(\sum_{l=1}^{\infty} \tilde{k}_{r p}^{(l)} e^{j l \omega_{m} t}+c . c .\right), \\
\hat{f}_{e s p}(t)=\bar{f}_{e s p}+\left(\sum_{l=1}^{\infty} \tilde{f}_{e s p}^{(l)} e^{j l \omega_{m} t}+c . c .\right), \\
\widehat{f}_{e r p}(t)=\bar{f}_{e r p}+\left(\sum_{l=1}^{\infty} \tilde{f}_{e r p}^{(l)} e^{j l \omega_{m} t}+c . c .\right) \\
\widehat{f}_{m s p}(t)=\bar{f}_{m s p}+\left(\sum_{l=1}^{\infty} \tilde{f}_{m s p}^{(l)} e^{j l \omega_{m} t}+c . c .\right), \\
\widehat{f}_{m r p}(t)=\bar{f}_{m r p}+\left(\sum_{l=1}^{\infty} \tilde{f}_{m r p}^{(l)} e^{j l \omega_{m} t}+c . c .\right),
\end{gathered}
$$

where c.c. stands for the complex conjugate of the preceding terms.

The eigenvalue problem associated with the linear free vibration of the PGTs is

$$
\left(\mathbf{K}_{b}+\mathbf{K}_{m 0}\right) \mathbf{v}_{i}=\omega_{i}^{2} \mathbf{M} \mathbf{v}_{i}
$$

where $\mathbf{v}_{i}$ are the eigenvectors and $\omega_{i}$ are the natural frequencies. Applying the modal transformation, $\mathbf{x}=\mathbf{V z}$, and let

$$
\begin{aligned}
\mathbf{C}_{v} & =\mathbf{V}^{T} \mathbf{C V}, \\
\mathbf{G}_{b} & =\mathbf{V}^{T} \mathbf{K}_{b} \mathbf{V}, \\
\mathbf{G}_{s p} & =\mathbf{V}^{T} \mathbf{K}_{s p} \mathbf{V}, \\
\mathbf{R}_{s p} & =\mathbf{V}^{T} \mathbf{D}_{s p}, \\
\mathbf{F}_{v t} & =\mathbf{V}^{T} \mathbf{F}_{t}, \\
\mathbf{F}_{v d} & =\mathbf{V}^{T} \bar{F}_{d}, \\
\mathbf{F}_{v m} & =\mathbf{V}^{T} \bar{F}_{m},
\end{aligned}
$$

where $\bar{F}_{d}$ are the mean parts of $\mathbf{F}_{d}(\mathbf{x}, t)$ with components $\bar{f}_{e s p}$ and $\bar{f}_{\text {erp }} . \bar{F}_{m}$ are the mean parts of $\mathbf{F}_{m}(\mathbf{x}, t)$ with components $\bar{f}_{m s p}$ and $\bar{f}_{\mathrm{mr} p}$ as presented in equation (20). The equation of motions can be written in the modal space as 


$$
\begin{aligned}
\ddot{\mathbf{z}}+ & \mathbf{C}_{v} \dot{\mathbf{z}}+\left\{\mathbf{G}_{b}+\sum_{p=1}^{N}\left[\bar{k}_{s p}+\left(\sum_{l=1}^{\infty} \tilde{k}_{s p}^{(l)} e^{j l \omega_{m} t}+c . c .\right) \Theta\left(\delta_{s p}\right) \mathbf{G}_{s p}+s \longrightarrow r\right]\right\} \mathbf{z} \\
& -\sum_{p=1}^{N}\left[\left(\sum_{l=1}^{\infty} \tilde{f}_{e s p}^{(l)} e^{j l \omega_{m} t}+c . c .\right) \Theta\left(\delta_{s p}\right) \mathbf{R}_{s p}+s \longrightarrow r\right] \\
& +\sum_{p=1}^{N}\left[\left(\sum_{l=1}^{\infty} \tilde{f}_{m s p}^{(l)} e^{j l \omega_{m} t}+c . c .\right) \Theta\left(\delta_{s p}\right) \mathbf{R}_{s p}+s \longrightarrow r\right]=\mathbf{F}_{v t}+\mathbf{F}_{v d}-\mathbf{F}_{v m}
\end{aligned}
$$

where $s \longrightarrow r$ represent the corresponding terms of $\mathrm{r}$-p meshes. From the results of modal analysis, one can find that the $1^{\text {st }}$ mode is rigid mode, the $2^{\text {nd }}$ and $(N+2)^{\text {th }}$ are rotational modes with distinct natural frequency, and the $3^{\text {rd }}$ to $(N+1)^{\text {th }}$ modes have equal natural frequency. It has been proved that, the vibration of PGTs system is mainly the superposition of these two rotational modes [34].

2.2. Problem Definition. In Figure 10, the time histories of $\mathrm{s}$-p mesh forces of a 5-planet example system with given parameters in Table 1 are presented. Without any error, the mesh forces for all the s-p mesh pairs are equal. As shown in Figure 10(a), the value of mesh force at point $\mathrm{A}$ is the maximum. Let $f_{\delta s p}$ and $f_{\delta r p}$ denote the peak value of s-p and r-p mesh forces, respectively. With tooth thickness error on the $1^{\text {st }}$ gear mesh $\bar{E}_{p 1}=10 \mu \mathrm{m}$, the corresponding time histories of s-p mesh forces are shown in Figure 10(b). It is clear that the $1^{\text {st }} \mathrm{s}$-p mesh force $f_{\delta s 1}$ is dramatically increased and $f_{\delta s 2}=f_{\delta s 5}, f_{\delta s 3}=f_{\delta s 4}$.

The ideal load condition for a PGT system is that each path will carry an equal load and the dynamic mesh force values are low. To illustrate the dynamic load sharing, the load sharing coefficients of the s-p mesh $L_{s p}$ and r-p mesh $L_{r p}$ are defined as

$$
\begin{aligned}
& L_{s p}=\frac{N f_{\delta s p}}{\sum_{n=1}^{N} f_{\delta s n}}, \\
& L_{r p}=\frac{N f_{\delta r p}}{\sum_{n=1}^{N} f_{\delta r n}}, \quad p=1, \cdots, N .
\end{aligned}
$$

The s-p load sharing factor $L_{s}$ and the r-p load sharing factor $L_{r}$ are defined as

$$
\begin{aligned}
L_{s} & =\max \left(L_{s p}\right), \\
L_{r} & =\max \left(L_{r p}\right), \quad p=1, \ldots, N .
\end{aligned}
$$

In order to indicate the maximum mesh force, the dynamic load factor $\Gamma$ is defined as

$$
\begin{gathered}
\Gamma=\max \left(\Gamma_{s}, \Gamma_{r}\right), \\
\Gamma_{s}=\frac{\max \left(f_{\delta s p}\right)}{\left(T_{s} / r_{s} / N\right)}, \\
\Gamma_{r}=\frac{\max \left(f_{\delta r p}\right)}{\left(T_{s} / r_{s} / N\right)} .
\end{gathered}
$$

In the case of the PGTs manufacture perfectly without error, as shown in Figure 10(a), $L_{s}=1$ and $\Gamma=1.055$. In the case of $\bar{E}_{p 1}=10 \mu \mathrm{m}$, as shown in Figure 10(b), $L_{s}=1.13$ and $\Gamma=1.19$. It is clear that the error causes uneven mesh forces and the increasement of the maximum mesh forces.

\section{Perturbation Analysis on Dynamic Mesh Forces}

To investigate the dynamic load sharing and load factor of PGTs, an approximate solution for the mesh force is sought by using the MMS [35]. The tooth separation function can be approximated as

$$
\Theta_{s p}=1+\sum_{l=0}^{\infty} \widetilde{\theta}_{s p}^{(l)} e^{j l\left(\omega_{m} t_{0}-\phi_{s p}\right)}+c . c .
$$

The approximated expression of $\Theta_{r p}$ is similar to that of $\Theta_{s p}$. The phases $\phi_{s p}$ and $\phi_{r p}$ will be chosen subsequently such that the tooth separation is in-phase with the mesh deflection. Similar to the procedure as presented in references $[24,27]$, an expression of the response amplitude $a_{i}$ and mesh frequency $\omega_{m}$ can be obtained as 


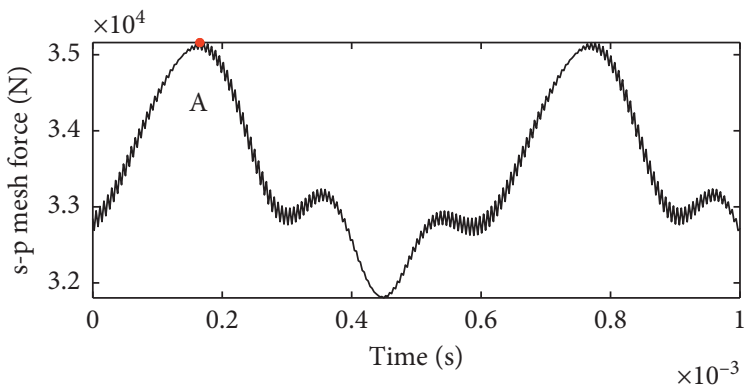

(a)

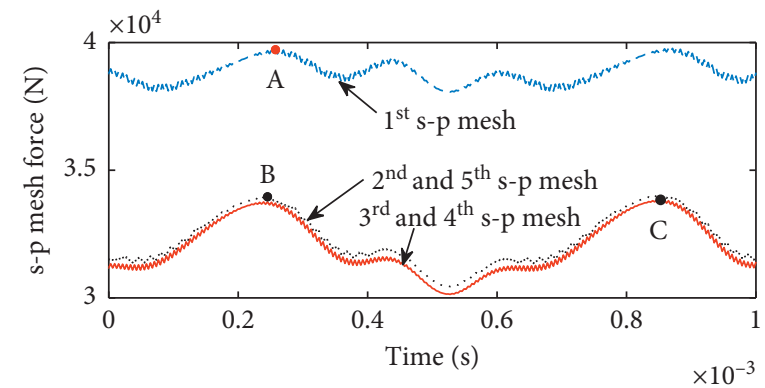

(b)

Figure 10: Time history of s-p mesh force for a 5-planet system. (a) Without errors; (b) $e_{r 1}=e_{s 1}=10 \mu \mathrm{m}$.

TABLE 1: System parameters of the example PGTs.

\begin{tabular}{lc}
\hline Parameters & Values \\
\hline No. of planets, $N$ & $3 \sim 6$ \\
Support stiffness of sun gear $(\mathrm{N} / \mathrm{m})$ & $1 \mathrm{e} 10$ (fixed), 1e6 \\
(float) \\
Mean s-p mesh stiffness $(\mathrm{N} / \mathrm{m})$ & $0.62 \mathrm{e} 9$ \\
Mean r-p mesh stiffness $(\mathrm{N} / \mathrm{m})$ & $0.85 \mathrm{e} 9$ \\
First harmonic of s-p mesh stiffness $(\mathrm{N} / \mathrm{m})$ & $0.14 \mathrm{e} 9$ \\
First harmonic of $\mathrm{r}-\mathrm{p}$ mesh stiffness $(\mathrm{N} / \mathrm{m})$ & $0.11 \mathrm{e} 9$ \\
s-p, r-p mesh phasing angle & 0 \\
Pressure angle $(\mathrm{deg})$ & 22.5 \\
Input torque to sun, $T_{s}(\mathrm{Nm})$ & 1130 \\
Sun inertia $\left(J_{s} / r_{s}^{2}\right)(\mathrm{kg})$ & 6.21 \\
Planet inertia $\left(J_{p} / r_{p}^{2}\right)(\mathrm{kg})$ & 4.89 \\
Damping ratio & 0.02 \\
Tooth number of sun gear in 5-planet & 34 \\
system & \\
Tooth number of planet gear in 5-planet & 31 \\
system & 96 \\
Tooth number of ring in 5-planet system & 4 \\
Module (mm) & 40 \\
Face width (mm) &
\end{tabular}

$$
\begin{aligned}
\omega_{m}= & \omega_{i}+\frac{1}{2 \omega_{i} a_{i}}\left[\Xi_{1} a_{i}+2 \Xi_{2} \pm 2 \sqrt{\Xi_{3}^{2}-\left(\omega_{i}^{2} a_{i} \zeta_{i}\right)^{2}}\right], \\
\Xi_{1}= & \sum_{p=1}^{N}\left|\bar{k}_{s p}\right|\left[\tilde{\theta}_{s p}^{(0)}+\tilde{\theta}_{s p}^{(2)}\right] G_{s p i i}+s \longrightarrow r, \\
\Xi_{2}= & \sum_{w=1}^{N+3} \sum_{p=1}^{N}\left|\bar{k}_{s p}\right| G_{s p i w} \tilde{\theta}_{s}^{(1)}\left(\frac{f_{t w}-f_{m w}+f_{d w}}{\omega_{w}^{2}}\right) \\
& -\sum_{p=1}^{N} \tilde{\theta}_{s}^{(1)}\left(\bar{f}_{d s p}-\bar{f}_{m s p}\right) R_{s p i}+s \longrightarrow r, \\
\Xi_{3}= & \sum_{w=1}^{N+3} \sum_{p=1}^{N} \tilde{k}_{s p}^{(1)}\left(\frac{f_{t w}-f_{m w}+f_{d w}}{\omega_{w}^{2}}\right) G_{s p i w} \\
& -\sum_{p=1}^{N}\left(\tilde{f}_{d s p}^{(1)}-\tilde{f}_{m s p}^{(1)}\right) R_{s p i}+s \longrightarrow r,
\end{aligned}
$$

where $f_{t w}, f_{d w}$, and $f_{m w}$ are the $w$ th element of the vectors $\mathbf{F}_{v t}$ $\mathbf{F}_{v d}$, and $\mathbf{F}_{v m}$, respectively. $G_{s p i w}$ is the $(i, w)$ elements of $\mathbf{G}_{s p}$ and $R_{s p i}$ is the $i$ th elements of $\mathbf{R}_{s p} . \zeta_{i}$ is the damping ratio. The dynamic mesh forces include the static components and timevarying components. The peak values of the time-varying s-p and $\mathrm{r}-\mathrm{p}$ mesh forces can be expressed as

$$
\begin{aligned}
& f_{\delta s p}=\frac{T_{s}}{r_{s} N}+\bar{k}_{s p}\left|v_{\delta s p}\right| a_{i}+\bar{k}_{s p} h_{s p}-\bar{k}_{s p} e_{s p}, \\
& f_{\delta r p}=\frac{T_{s}}{r_{s} N}+\bar{k}_{r p}\left|v_{\delta r p}\right| a_{i}+\bar{k}_{r p} h_{r p}-\bar{k}_{r p} e_{r p},
\end{aligned}
$$

where $v_{\delta s p}$ and $v_{\delta r p}$ are two coefficients associated with the modal vectors $\mathbf{V}$ and can be expressed as

$$
\begin{aligned}
& v_{\delta s p}=-v_{c} \cos \alpha_{s}-v_{x s} \sin \psi_{s p}+v_{y s} \cos \psi_{s p}+v_{u s}+v_{p}, \\
& v_{\delta r p}=-v_{c} \cos \alpha_{r}+v_{r}-v_{p} .
\end{aligned}
$$

Substituting equation (29) into the definition of the dynamic load sharing coefficients in equation (24),

$$
L_{s p}=\frac{N\left[\bar{k}_{s p}\left|v_{\delta s p}\right| a_{i}+\bar{k}_{s p} h_{s p}+\left(T_{s} / r_{s} N\right)-\bar{k}_{s p} e_{s p}\right]}{N \bar{k}_{s p}\left|v_{\delta s p}\right| a_{i}+N \bar{k}_{s p} h_{s p}+\left(T_{s} / r_{s}\right)-\sum_{n=1}^{N}\left(\bar{k}_{s n} e_{s n}\right) .}
$$

Let $\Upsilon_{s}$ represent the equal parts among all the $s-p$ meshes:

$$
\Upsilon_{s}=\bar{k}_{s p} a b s\left(v_{\delta s p} a_{i}\right)+\bar{k}_{s p} h_{s p}+\frac{T_{s}}{r_{s} N} .
$$

Substituting equation (32) into equation (31), equation (31) could be simplified as

$$
L_{s p}=\frac{\Upsilon_{s}-\bar{k}_{s p} e_{s p}}{\Upsilon_{s}-(1 / N) \sum_{n=1}^{N} \bar{k}_{s n} e_{s n}} .
$$

From equation (33), one can see that the load sharing coefficients are determined by $\Upsilon_{s}$ and $e_{s p}$. $\Upsilon_{s}$ includes the effects of the transmit torque, tooth profile modification, and dynamic vibrations, and the equivalent tooth thickness error $e_{s p}$ includes the effects of both tooth thickness errors and the pin position errors. 
Substituting equation (29) into the definition of the dynamic load factor in equation (26), one has

$$
\begin{aligned}
\Gamma_{s} & =\frac{\left(T_{s} / r_{s}\right)+N \bar{k}_{s p}\left|v_{\delta s p}\right| a_{i}+N \bar{k}_{s p} h_{s p}-\bar{k}_{s p} e_{s p}}{\left(T_{s} / r_{s}\right)} \\
& =1+\frac{N \bar{k}_{s p}\left|v_{\delta s p}\right| a_{i}+N \bar{k}_{s p} h_{s p}-\bar{k}_{s p} e_{s p}}{\left(T_{s} / r_{s}\right) .}
\end{aligned}
$$

The corresponding expressions for $r-p$ meshes are similar to the expressions for $\mathrm{s}-\mathrm{p}$ meshes.

\section{Results and Discussions}

In this investigation, the effects of the errors, the support stiffness of sun gear, the applied torque, and tooth profile modification on the load sharing factors and the dynamic load factors will be discussed following. The main parameters of the example systems with different number of planet gear are listed in Table 1. As shown in Table 1, the only difference among the given several PGT systems is the number of planet gears. The two natural frequencies associated with the first two rotational vibration modes, $\omega_{r 1}$ and $\omega_{r 2}$, are listed in Table 2 .

4.1. Validation of the Proposed Method. To verify the proposed method, assume the $1^{\text {st }}$ planet gear has the timeinvariant tooth profile errors, $e_{r 1}=e_{s 1}=50 \mu \mathrm{m}$, and the other components are perfectly manufactured and assembled for the examined PGTs. It should be noted that these equivalent tooth thickness errors on the $1^{\text {st }}$ planet may come from either the tooth thickness error or the pin position errors of the $1^{\text {st }}$ planet gear. The comparisons of the load coefficients obtained by MMS and NI with varying number of planet gear are shown in Figures 11 and 12. The loads on $\mathrm{r}-\mathrm{p}$ meshes are mainly concerned with $\omega_{m} \approx \omega_{r 1}$, while the loads on s-p meshes are mainly concerned with $\omega_{m} \approx \omega_{r 2}$.

With sun gear fixed, as shown in Figure 11 , the $1^{\text {st }}$ planet gear takes the heaviest load due to the errors. Because of the symmetry positions to the $1^{\text {st }}$ planet gear, the $2^{\text {nd }}$ and $N^{\text {th }}$ planet gears carry equal loads; the $3^{\text {rd }}$ and $(N-1)^{\text {th }}$ planet gears carry equal loads. With sun gear floated, as shown in Figure 12, all the planet gears in 3-planet PGT systems carry almost equal load and the opposite planet gears in 4-planet gears carry equal load. With the increase of the number of planet gears, the load sharing coefficients become more sensitive to the errors. These conclusions agree well with the conclusions obtained by previous static analysis $[6,7,10]$. In Figures 11 and 12, all the curves obtained by MMS agree well with the NI results.

One can find the dynamic load sharing coefficients vary with the changing of mesh frequency. This is because the amplitudes of mesh forces are the functions of the modal vibration amplitudes $a_{i}$, which is associated with the mesh frequency, as expressed in equation (28). The interesting phenomenon is that, with $\omega_{m} \approx \omega_{r 1}, \omega_{r 2}$, the load sharing conditions are improved. This is because the vibration
TABLE 2: Natural frequencies associated with the first two rotational modes.

\begin{tabular}{lc}
\hline No. of planets, $N$ & $\omega_{r 1}$ and $\omega_{r 2}(\mathrm{~Hz})$ \\
\hline 3 & 2114,4674 \\
4 & 2233,5249 \\
5 & 2329,5775 \\
6 & 2412,6260 \\
\hline
\end{tabular}

amplitudes are increased, and the dynamic mesh forces caused by the vibration are the dominant factors in the primary resonance ranges. Consequently, the effects of errors are relatively decreased, and the load sharing coefficient associated with the $1^{\text {st }}$ planet gear is decreased. However, with this improvement of the load sharing, the dynamic load conditions get worse, as show in Figure 13. The dynamic load factors dramatically increase near the primary resonance due to the large vibrations. Under dynamic working conditions, both of the load sharing factors and dynamic load factors are considered to evaluate the load conditions among all the planet gears.

It should be noted that, the softening nonlinearity and vibration jump phenomenon appear in the primary resonance ranges. The frequency-load sharing coefficient curves have three branches near the primary resonance, and the middle branch is unstable.

4.2. Effects of the Errors in Planet Gears. It is believed that both of the planet pin position errors and tooth thickness errors are parts of the vibration excitations of PGTs $[31,32]$. In order to explore the effects of tooth thickness errors and pin position errors of planet gear on the planet load sharing, especially on the dynamic load factor, the effects of the equivalent tooth thickness error on the load sharing factors and dynamic load factors are discussed here.

Figure 14 shows the effects of $e_{r 1}$ and $e_{s 1}$ for the 5-planet system versus mesh frequency. With the increasing of $e_{r 1}$ and $e_{s 1}$, the load sharing factors increase and the frequency ranges with contact loss expanded. It is worth noting that the increase of the load sharing factors is almost proportional to the increase of $e_{r 1}$ and $e_{s 1}$, as shown in Figure 15. The results in Figure 15(a) have similar trend with the results presented in references $[9,15]$. According to Bodas and Kahraman [6], the load sharing is proportional to the absolute magnitude of carrier and gear manufacturing errors. From Figure 15(b), one can see that, in dynamic conditions, the load sharing is also proportional to the absolute magnitude of equivalent tooth thickness errors. However, with same amount of equivalent tooth thickness error, the load sharing in dynamic conditions is better.

It is believed that the error induces vibration and then leads the increase of the dynamic load factor, as shown in Figure 16. In addition, by this figure, one can find the dynamic load factor is proportional to the equivalent tooth thickness error. Selecting high-precision gears will certainly reduce the dynamic mesh load and improve load sharing performance. 


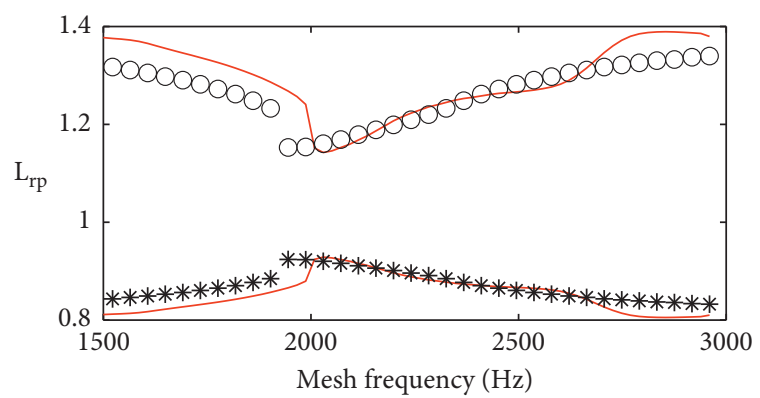

(a)

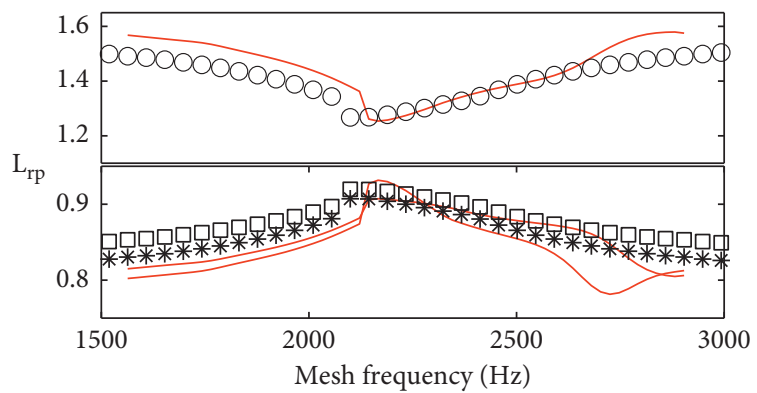

(c)

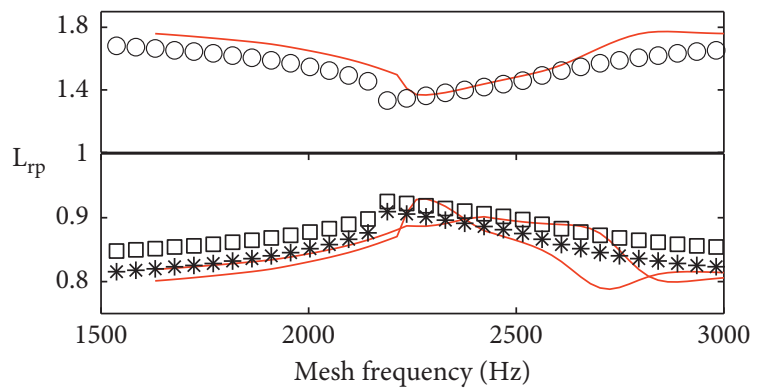

(e)

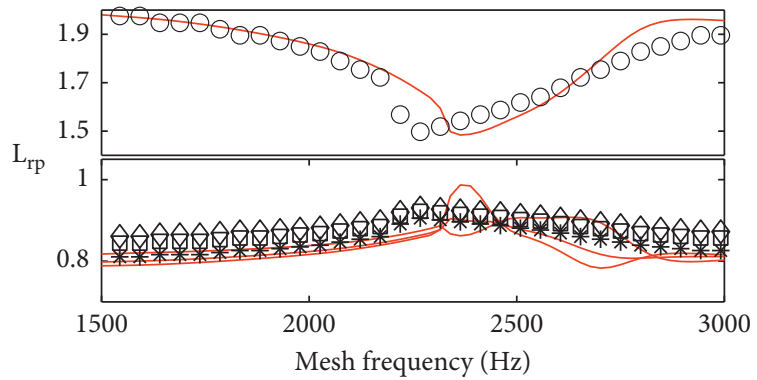

(g)

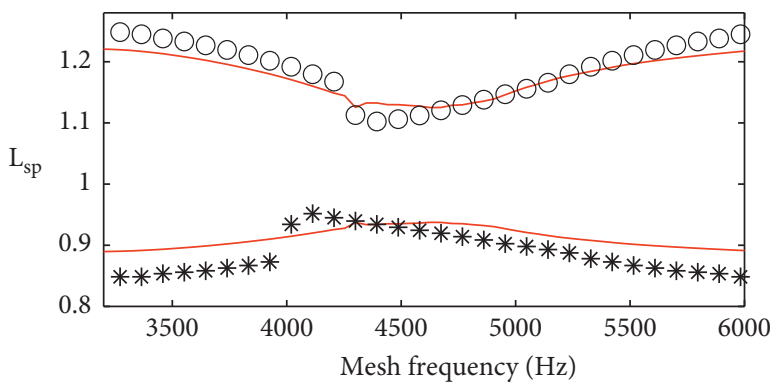

(b)

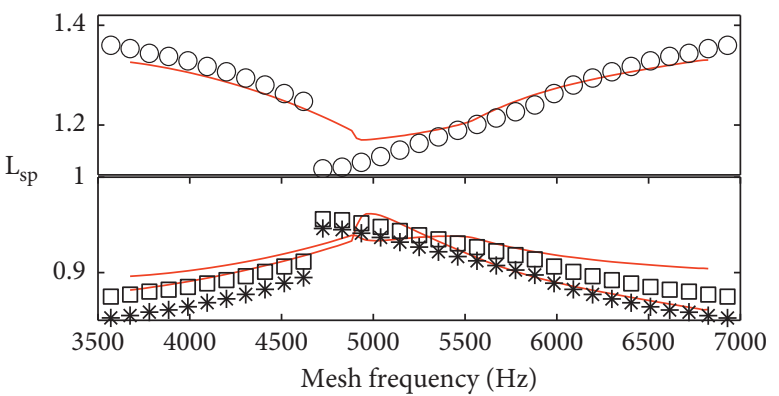

(d)

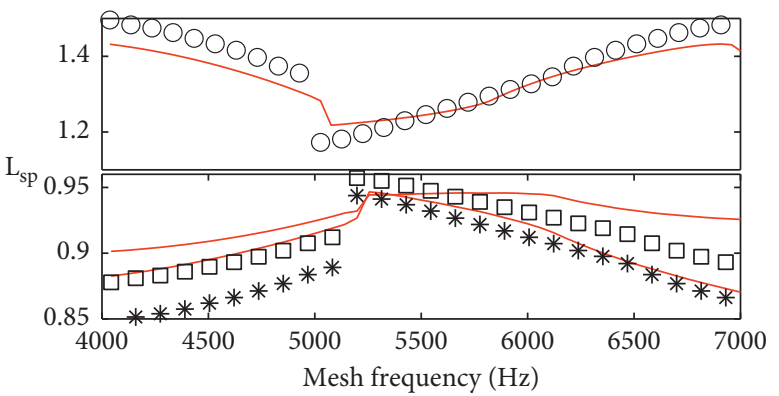

(f)

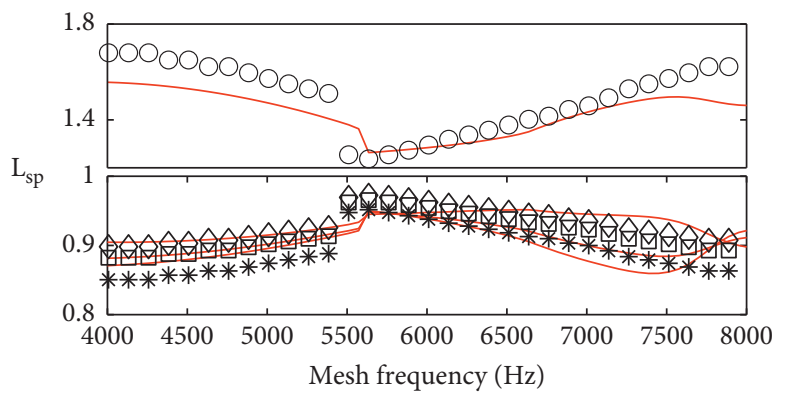

(h)

FIgURE 11: Load sharing coefficients with fixed sun gear. (ooo), the 1st planet; $(* * *)$, the $2^{\text {nd }}$ and the $(N)^{\text {th }}$ planets; ( $\left.\square \square \square\right)$, the $3^{\text {rd }}$ and the $(N-1)^{\text {th }}$ planets; $(\diamond \diamond \diamond)$, the $4^{\text {th }}$ planet; $(-)$, NI results. (a) r-p meshes of 3-planet system with $\omega_{m} \approx \omega_{r 1}$; (b) s-p meshes of 3-planet system with $\omega_{m} \approx \omega_{r 2}$; (c) r-p meshes of 4-planet system with $\omega_{m} \approx \omega_{r 1}$; (d) s-p meshes of 4-planet system with $\omega_{m} \approx \omega_{r 2}$; (e) r-p meshes of 5-planet system with $\omega_{m} \approx \omega_{r 1}$; (f) s-p meshes of 5-planet system with $\omega_{m} \approx \omega_{r 2} ;(\mathrm{g})$ r-p meshes of 6-planet system with $\omega_{m} \approx \omega_{r 1}$; (h) s-p meshes of 6-planet system with $\omega_{m} \approx \omega_{r 2}$.

4.3. Effects of Pin Position Error of Central Components. Pin position error of central components is a critical factor affecting the dynamic load sharing. In Figure 17, the load sharing coefficients for a 5-planet system with either $\bar{E}_{c s}=10$ $\mu m$ or $\bar{E}_{c r}=10 \mu m$ along the $x$-direction are shown. Under the effects of the pin position error of sun gear, the load of the $1^{\text {st }}$ planet gear is maximum. This is because the $1^{\text {st }}$ planet is the nearest one to the sun gear due to the pin position error. This pin position error has unequal effects on each planet gear, because of the different angles between the mesh lines and the direction of the pin position errors. The same pin position error of ring gear has opposite effects on the 


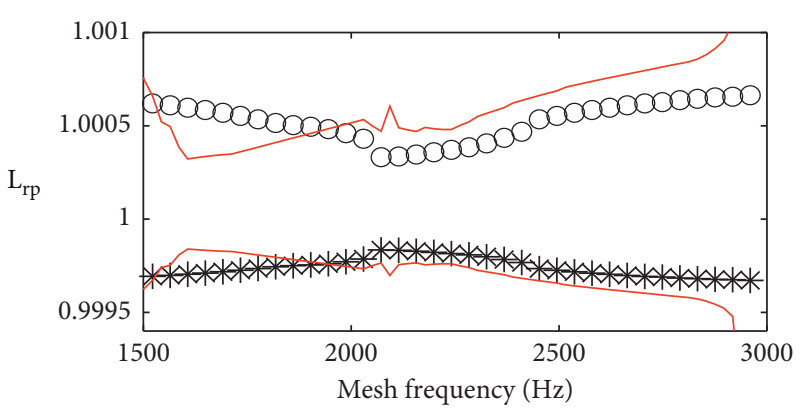

(a)

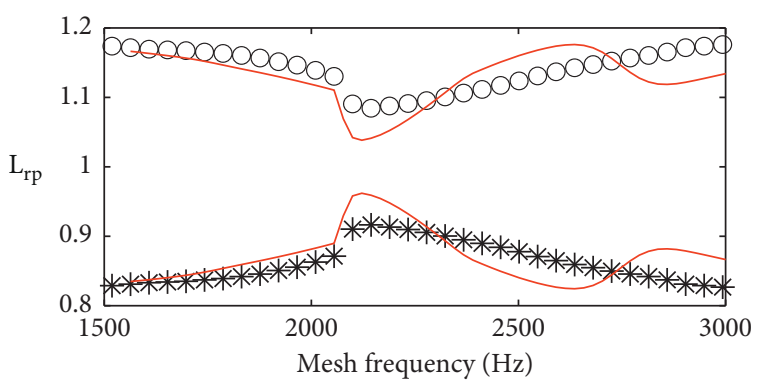

(c)

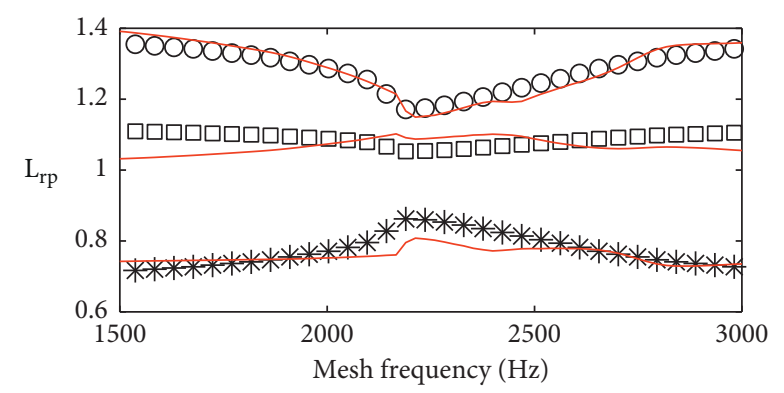

(e)

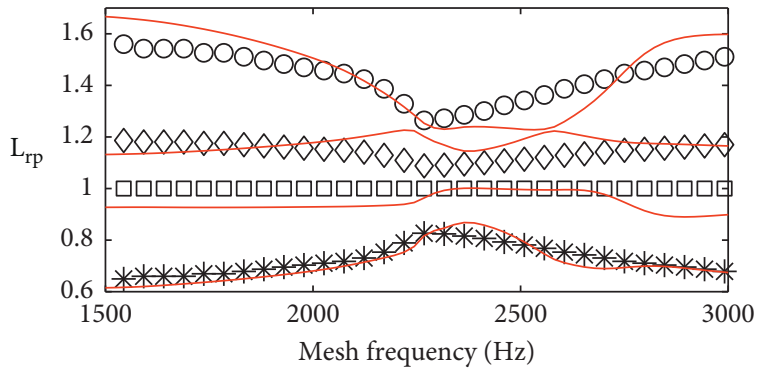

(g)

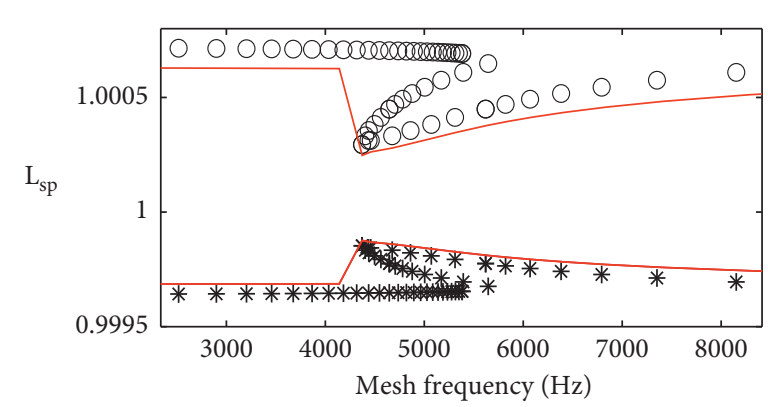

(b)

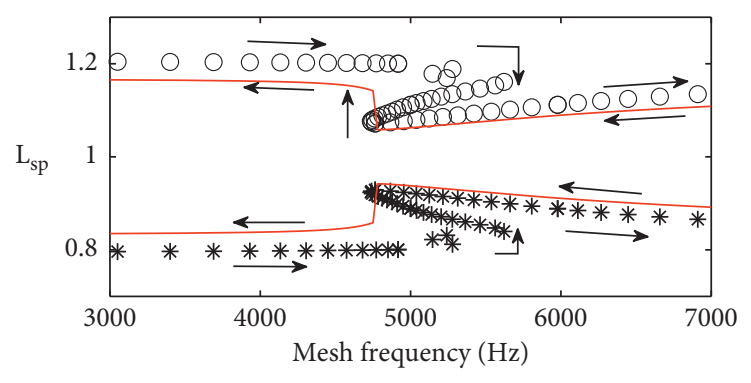

(d)

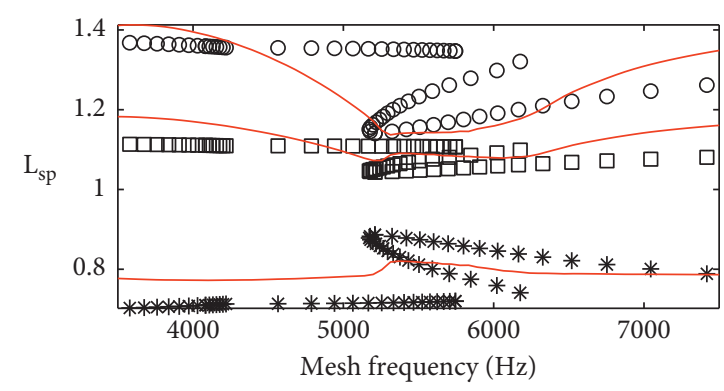

(f)

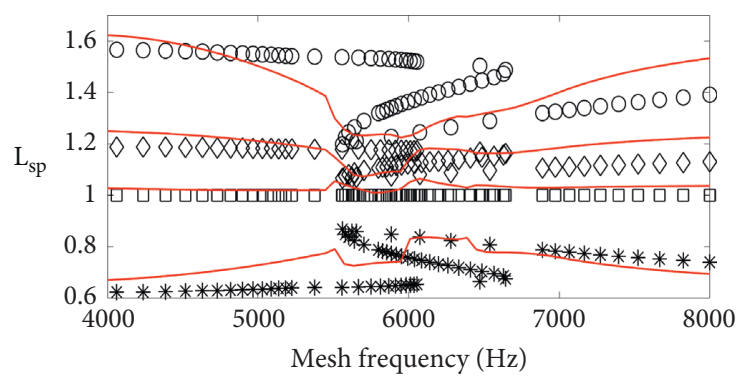

(h)

FIGURE 12: Load sharing coefficients with floating sun gear. (ooo), the 1 st planet; $(* * *)$, the $2^{\text {nd }}$ and the $(N)^{\text {th }}$ planets; ( $\left.\square \square \square\right)$, the $3^{\text {rd }}$ and the $((\mathrm{N})-1)^{\text {th }}$ planets; $(\diamond \diamond \diamond)$, the $4^{\text {th }}$ planet; $(-)$, NI results. (a) r-p meshes of 3-planet system with $\omega_{m} \approx \omega_{r 1}$; (b) s-p meshes of 3-planet system with $\omega_{m} \approx \omega_{r 2} ;$ (c) r-p meshes of 4-planet system with $\omega_{m} \approx \omega_{r 1} ;$ (d) s-p meshes of 4-planet system with $\omega_{m} \approx \omega_{r 2}$; (e) r-p meshes of 5-planet system with $\omega_{m} \approx \omega_{r 1}$; (f) s-p meshes of 5-planet system with $\omega_{m} \approx \omega_{r 2} ;(\mathrm{g}) \mathrm{r}$-p meshes of 6-planet system with $\omega_{m} \approx \omega_{r 1}$; (h) s-p meshes of 6-planet system with $\omega_{m} \approx \omega_{r 2}$.

load sharing, as shown in Figures 17(c) and 17(d). The load of the $1^{\text {st }}$ planet is minimum. This is because the $1^{\text {st }}$ planet is furthest to the ring gear with this pin position error. These results also agree well with the conclusions obtained from static analysis in reference [19].

The effects of the pin position errors on dynamic load sharing factors versus mesh frequency are shown in Figure 18.
The load sharing factors are proportional to the pin position errors of sun gear and ring gear, and the frequency ranges with tooth separations are increased by the increasing of these errors. The curves in Figure 19 indicate that there exist little effects of the pin position errors on the dynamic factors. It suggests that the pin position errors of sun gear and ring gear affect the load sharing behaviours mainly as static factors. 


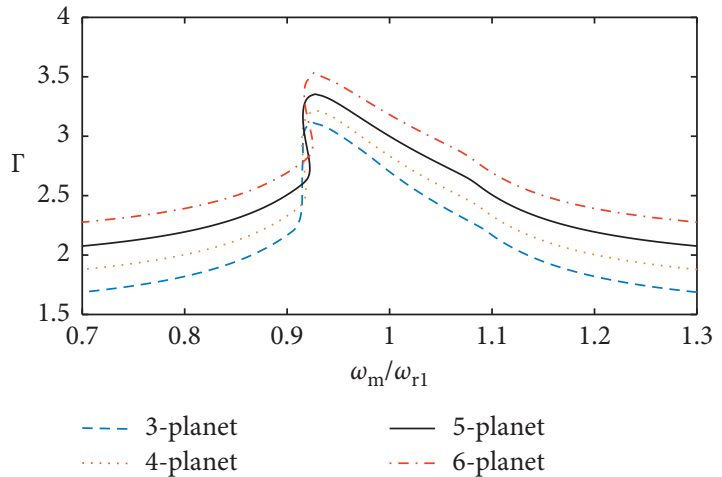

(a)

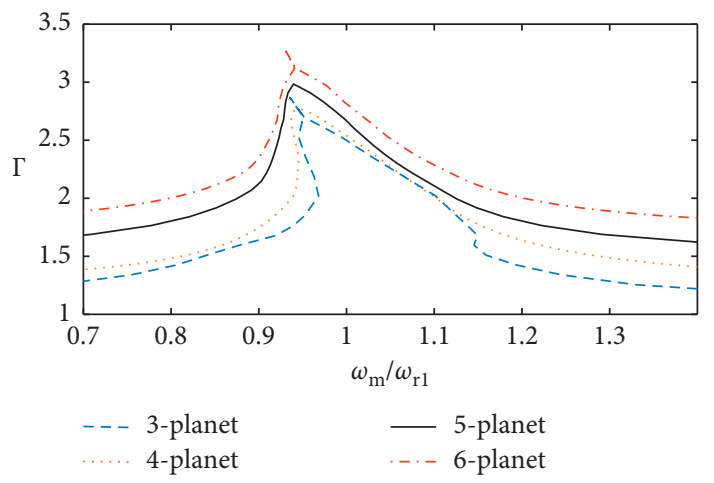

(c)

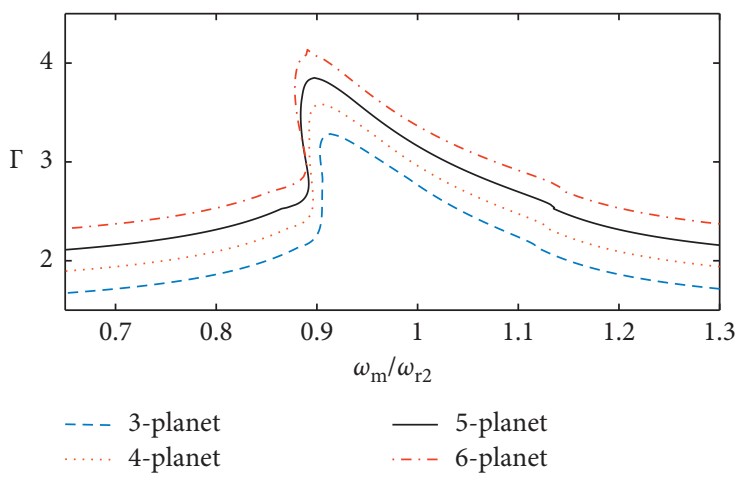

(b)

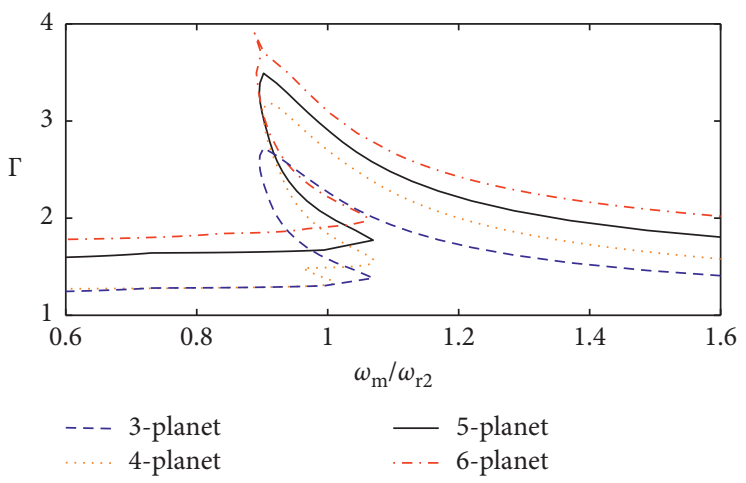

(d)

FIGURE 13: Dynamic load factors for PGT systems with $e_{r 1}=e_{s 1}=50 \mu \mathrm{m}$. (a) Fixed sun gear, $\omega_{m} \approx \omega_{r 1}$; (b) fixed sun gear, $\omega_{m} \approx \omega_{r 2}$; (c) float sun gear, $\omega_{m} \approx \omega_{r 1} ;$ (d) float sun gear, $\omega_{m} \approx \omega_{r 2}$.

4.4. Effects of Applied Torque. Applied torque is another key factor that affects the dynamic characteristic of PGT system. Figure 20 shows the effects of the applied torque on the dynamic factors versus mesh frequency with $e_{r 1}=e_{s 1}=50 \mu \mathrm{m}$. From Figure 20, one can see that heavy applied torque effectively decreases the dynamic load sharing factor. From equations (32) and (33), a reasonable explanation is that large torque increases the value of $\Upsilon_{s}$, and the effects of the errors on the load sharing factors are suppressed. Heavy applied torque may decrease the load sharing factors but increase the loads on all the meshes.

The load sharing coefficients of 5-planet system with varying applied torque in both quasistatic and dynamic conditions are shown in Figure 21. The curves in Figure 21(a) agree well with the results obtained under quasistatic conditions in reference [8]. Once again, the dynamic load sharing is better than the corresponding results in quasistatic, because of the effects of vibrations. Considering the static strength of gear tooth, increasing the applied torque to suppress the effects of equivalent tooth thickness error is not the first option.

4.5. Effects of the Support Stiffness of Sun Gear. In order to explore the mechanism of the float sun gear on the improvement of the dynamic load sharing, the comparisons of the translational displacements of sun gear of a five-planet system with/without float sun gear are shown in Figure 22.
With sun gear floated, the equilibrium position of sun gear is put away from the origin point, and the vibration amplitudes of the translational displacements are increased. From equation (29), one can find that float sun gear with low support stiffness allows the equilibrium position to change and induces the translational motions, which compensates the uneven effects of the equivalent tooth thickness errors.

In practical PGTs, the sun gear is not absolutely float or fixed. To further explore the effects of the sun gear support stiffness, the influences of the support stiffness on the load sharing factors and the dynamic load factor of sun gear for the 5-planet system are shown in Figures 23 and 24, respectively. The load sharing factor decreases with the support stiffness of sun gear decreasing, and the load sharing is improved. The dynamic load factor slightly decreases with the decreasing of the support stiffness of sun gear.

In the case of low rotational speed, the load sharing condition is close to those in quasistatic condition. As shown in Figure 25(a), the load sharing coefficients are almost constant when $k_{b s} \in\left[10^{6}, 10^{8}\right] \mathrm{N} / \mathrm{m}$. While $k_{b s}$ increases up to $\left[10^{8}, 10^{10}\right] \mathrm{N} / \mathrm{m}$, the load sharing coefficient of $1^{\text {st }}$ planet gear increases. These curves in Figure 25 agree well with the results presented in reference [9] in the case of quasistatic conditions. In dynamic conditions, as shown in Figure 25(b), the varying of load sharing coefficients with the support stiffness of sun gear has similar trend with the results in quasistatic conditions. While in dynamic load sharing, 


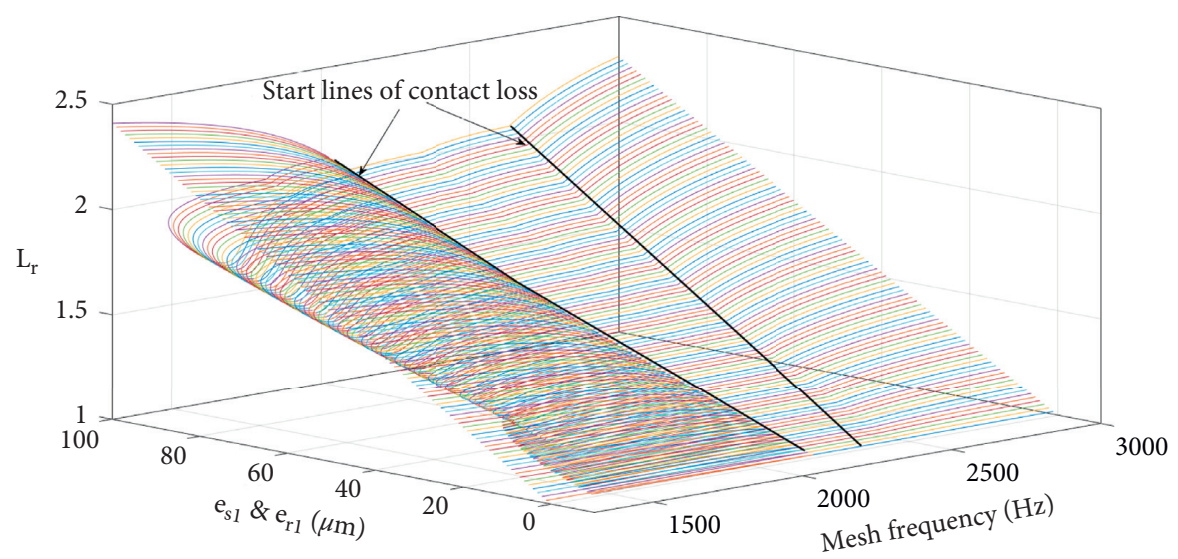

(a)

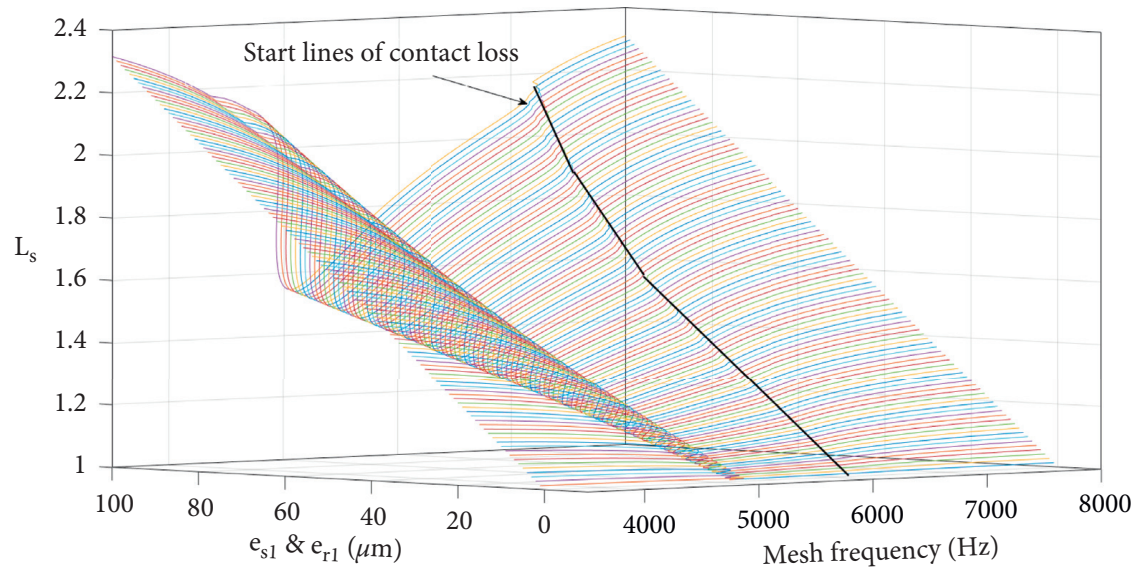

(b)

FIGURE 14: Load sharing coefficients versus mesh frequency for 5-planet system with varying $e_{r 1}, e_{s 1}$. (a) $\omega_{m} \approx \omega_{r 1}$; (b) $\omega_{m} \approx \omega_{r 2}$.

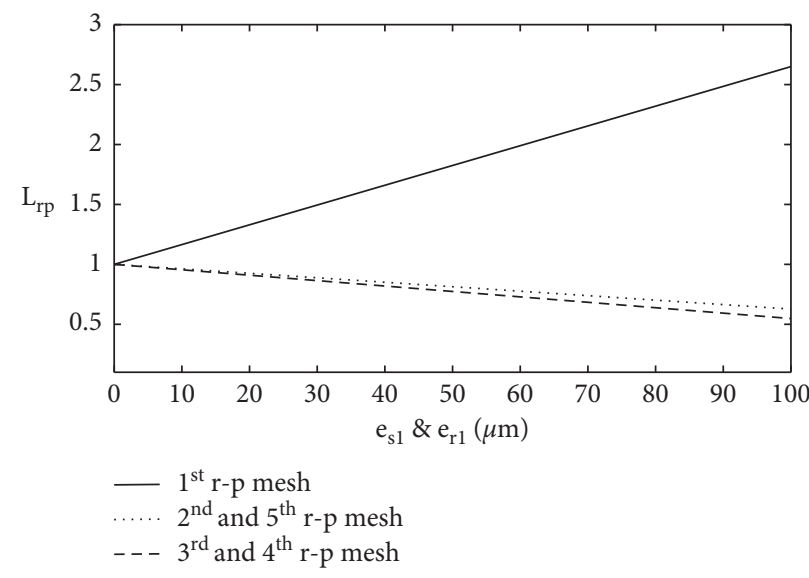

(a)

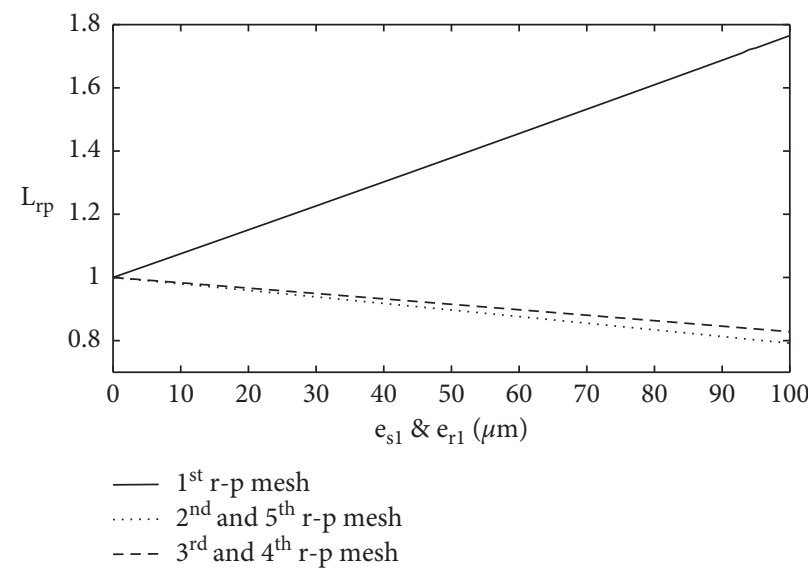

(b)

FIGURE 15: Load sharing coefficients for 5-planet system with varying $e_{r 1}, e_{s 1}$. (a) Quasistatic condition with low rotational speed; (b) sun gear rotational speed $4200 \mathrm{r} / \mathrm{min}$.

under the influence of system vibration, the $1^{\text {st }}$ planet gear load sharing coefficient is decreased and the load sharing is improved. As discussed above, floating sun gear decreases the dynamic mesh load and optimizes the load sharing, which is still one of the effective methods with high priority in dynamic conditions despite of larger sun vibrations. 


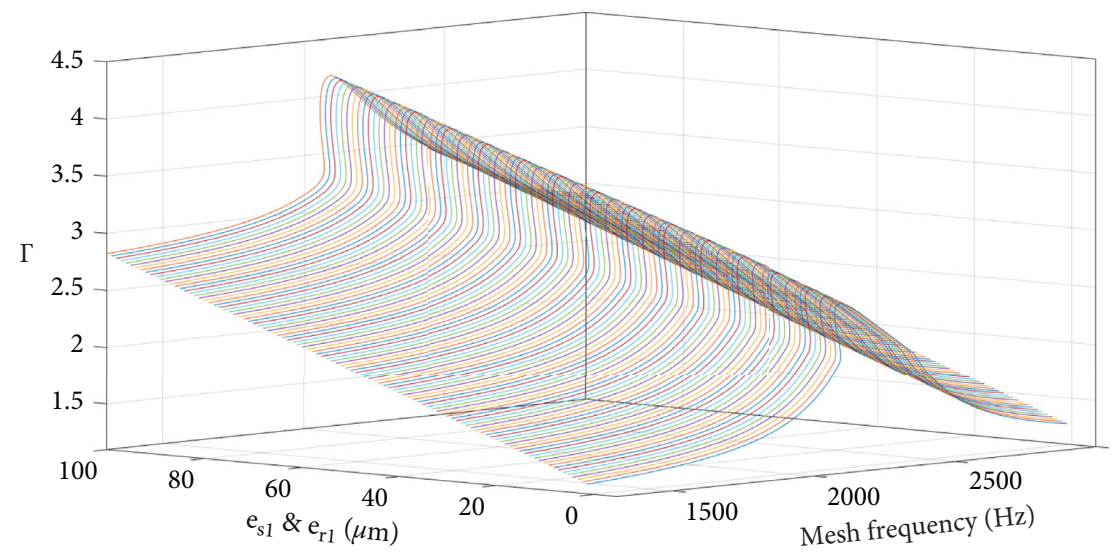

(a)

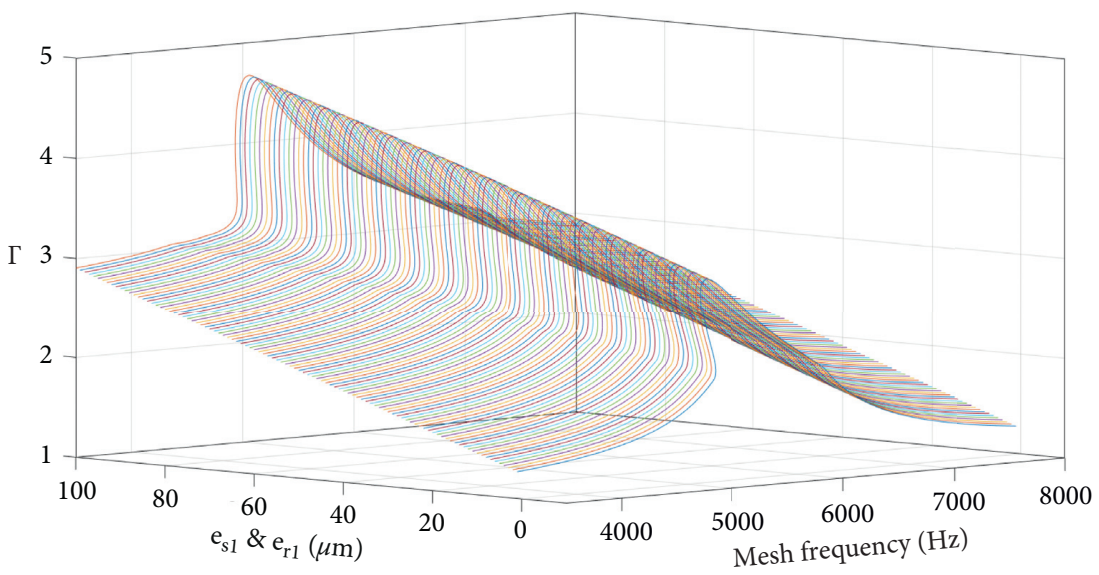

(b)

FIGURE 16: Dynamic load factors versus mesh frequency for 5-planet system with varying $e_{r 1}, e_{s 1}$. (a) $\omega_{m} \approx \omega_{r 1}$; (b) $\omega_{m} \approx \omega_{r 2}$.

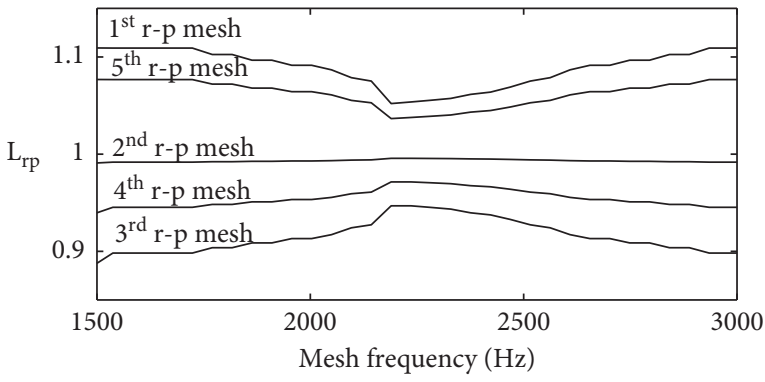

(a)

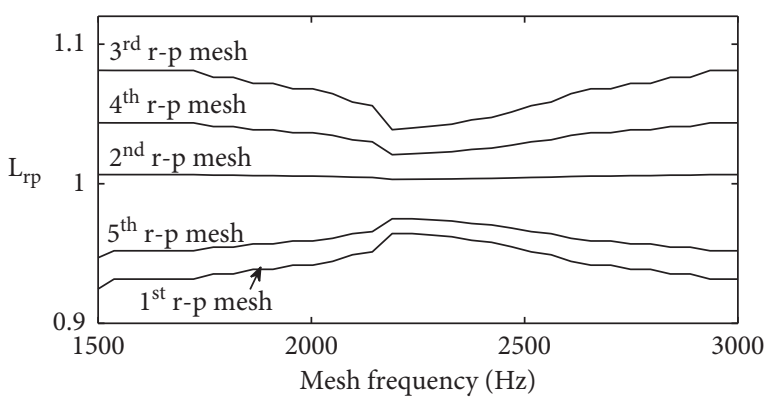

(c)

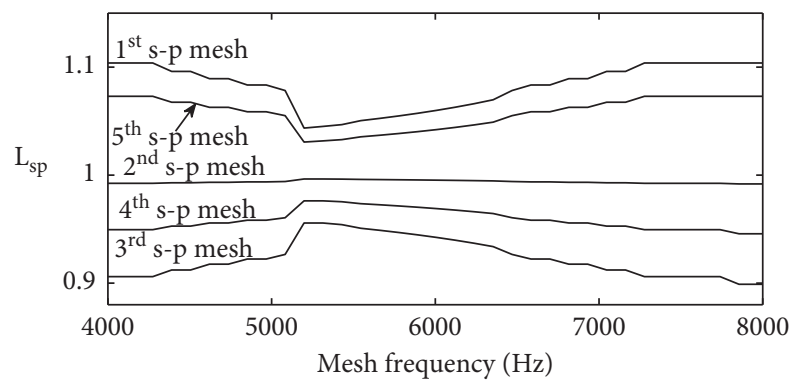

(b)

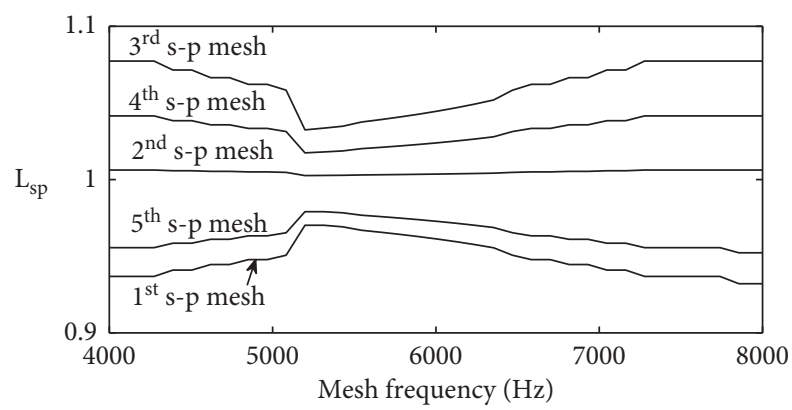

(d)

FIGURE 17: Effects of the pin position errors on the dynamic load coefficients. (a) $L_{r p}$ with $\bar{E}_{c s}=10 \mu \mathrm{m}$; (b) $L_{s p}$ with $\bar{E}_{c s}=10 \mu \mathrm{m}$; (c) $L_{r p}$ with $\bar{E}_{c r}=10 \mu \mathrm{m}$; (d) $L_{s p}$ with $\bar{E}_{c r}=10 \mu \mathrm{m}$. 


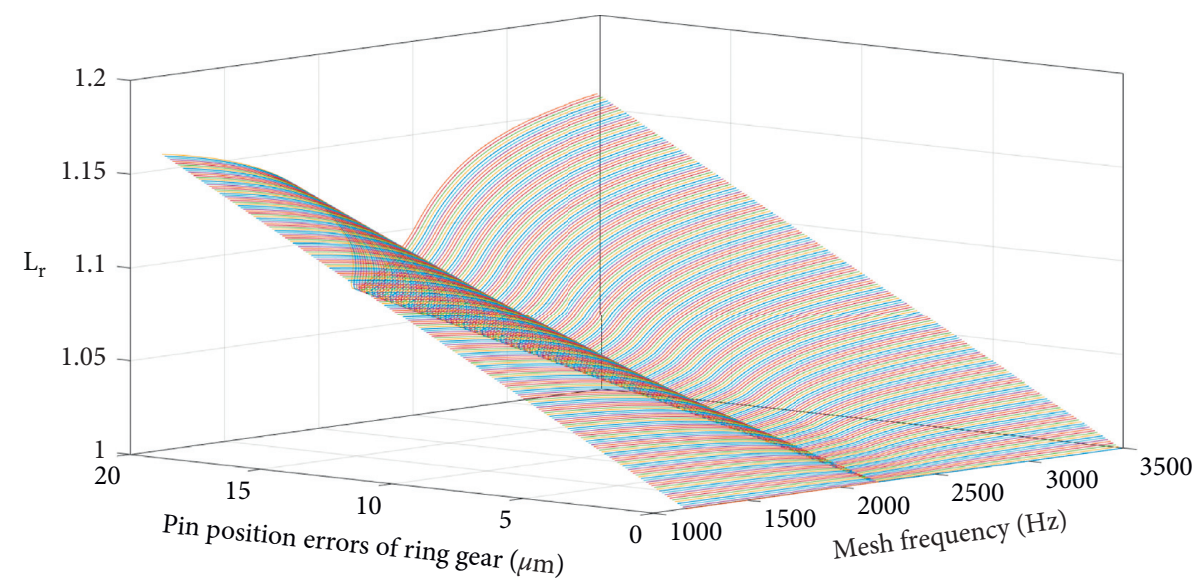

(a)

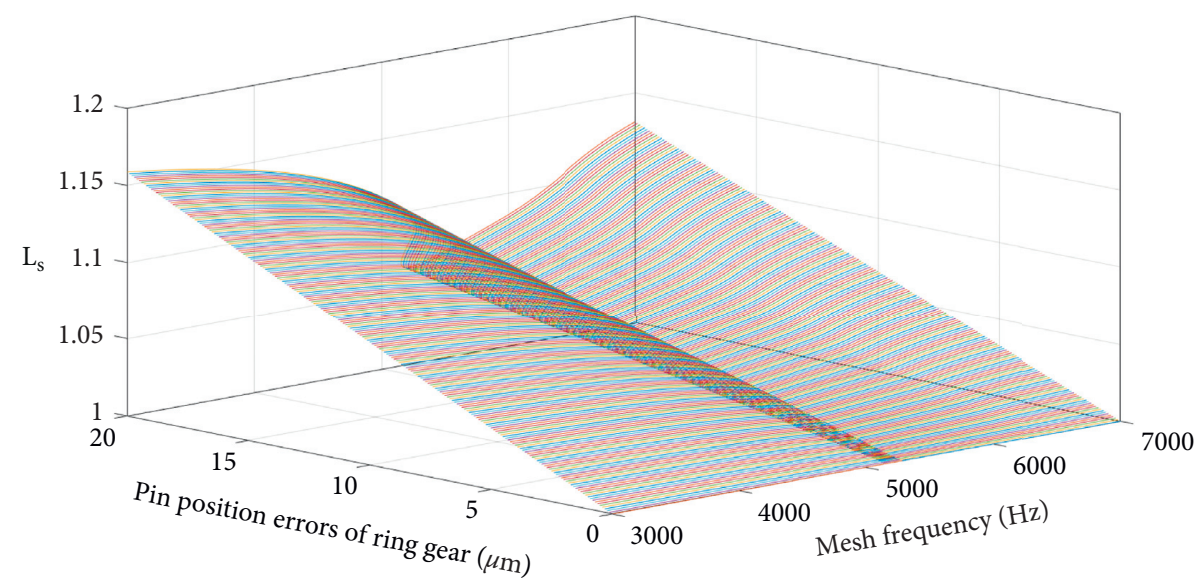

(b)

FIGURE 18: Effects of the pin position errors on the load sharing factors. (a) $\omega_{m} \approx \omega_{r 1}$; (b) $\omega_{m} \approx \omega_{r 2}$.

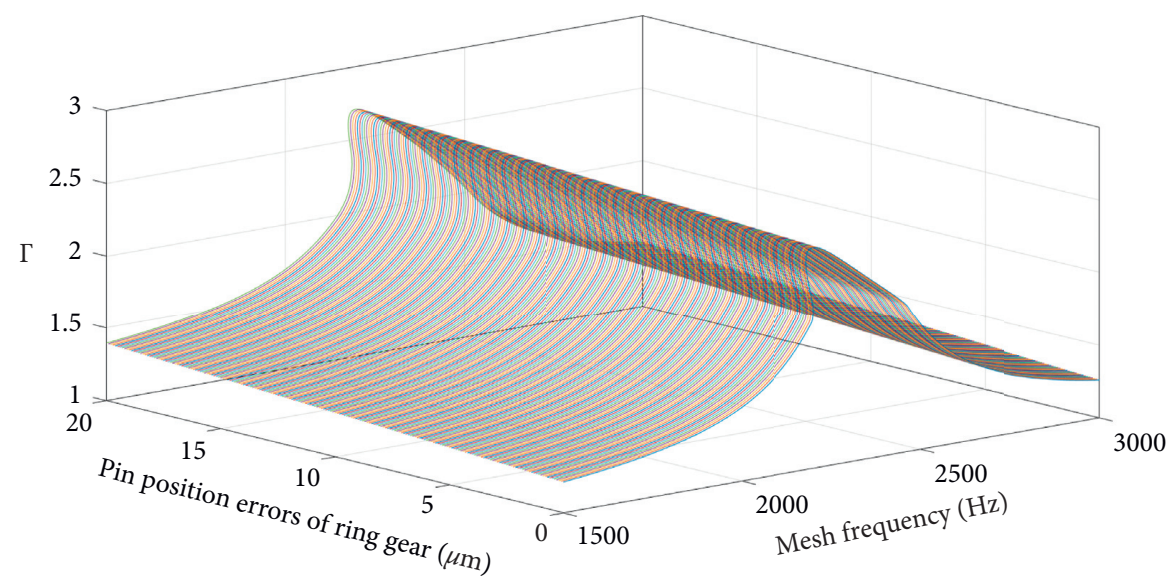

(a)

FIgURe 19: Continued. 


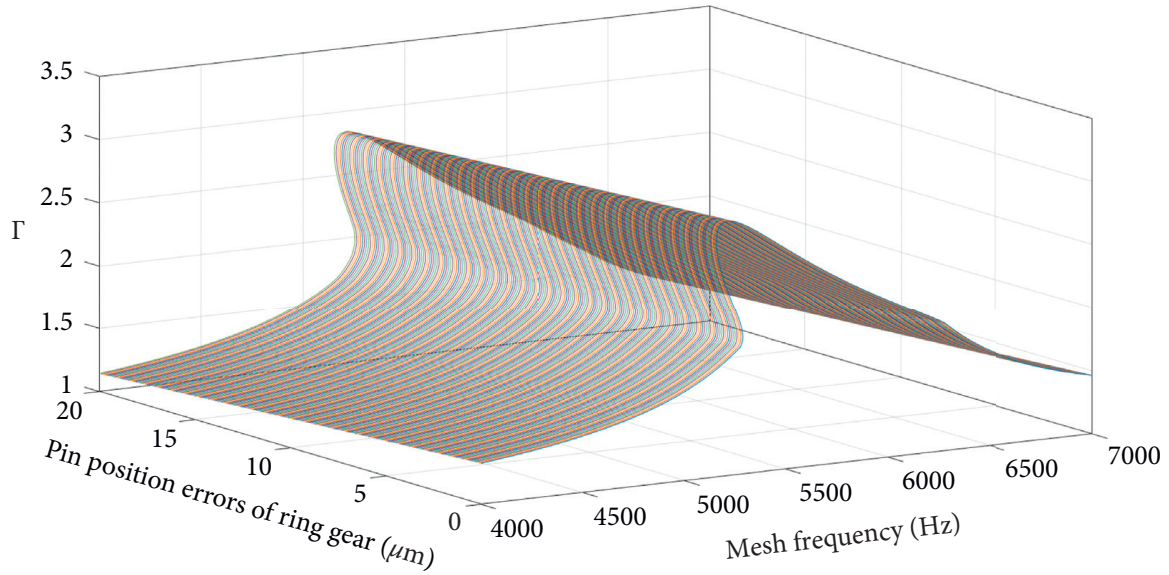

(b)

FIGURE 19: Effects of the pin position errors on the dynamic load factors. (a) $\omega_{m} \approx \omega_{r 1}$; (b) $\omega_{m} \approx \omega_{r 2}$.

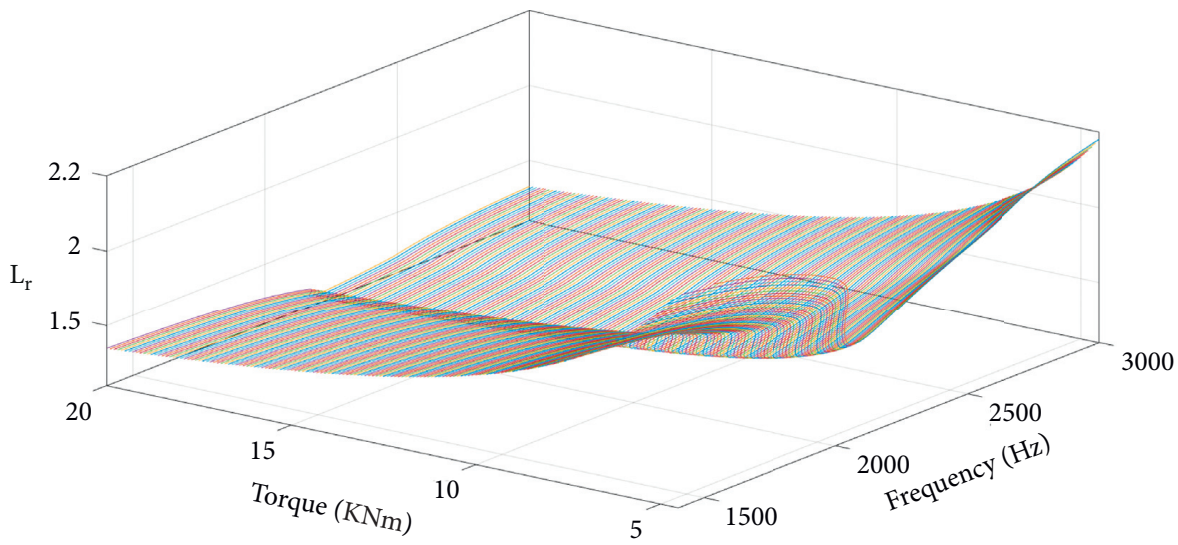

(a)

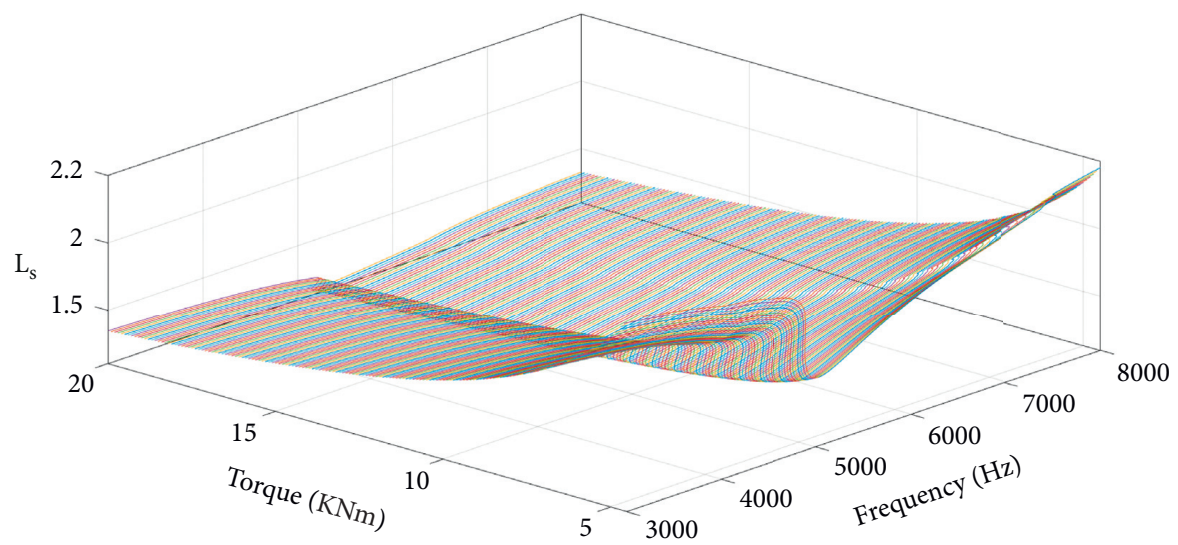

(b)

FIGURE 20: Load sharing factors for 5-planet system with varying applied torque. (a) $\omega_{m} \approx \omega_{r 1}$; (b) $\omega_{m} \approx \omega_{r 2}$. 


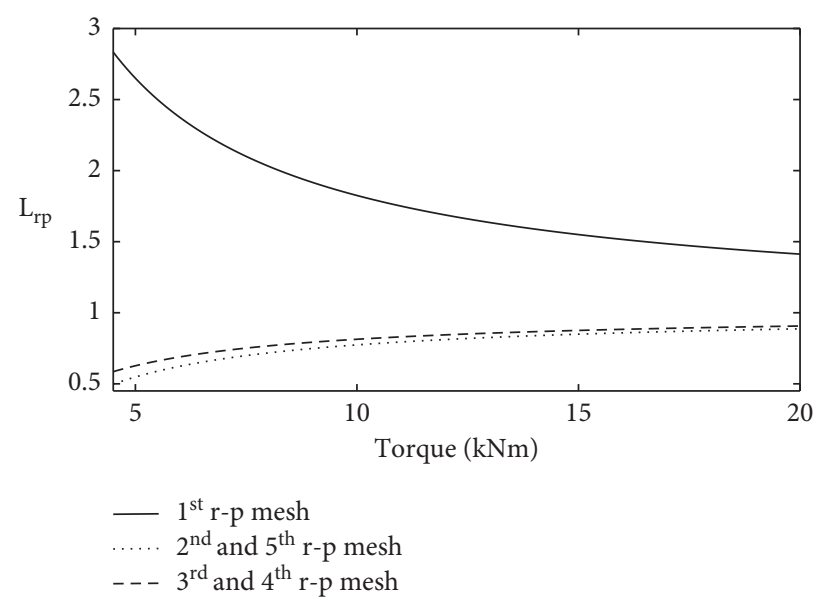

(a)

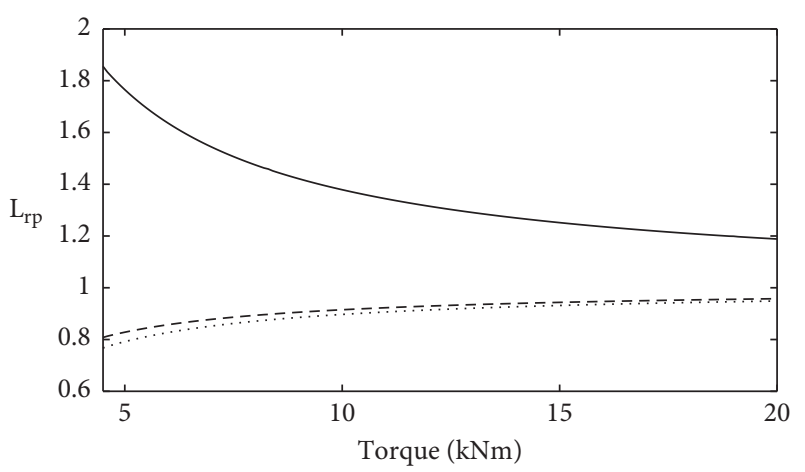

- $1^{\text {st }}$ r-p mesh

$\cdots 2^{\text {nd }}$ and $5^{\text {th }} r-p$ mesh

$--3^{\text {rd }}$ and $4^{\text {th }}$ r-p mesh

(b)

FIGURE 21: Load sharing coefficients for 5-planet system with varying applied torque. (a) Quasistatic condition with low rotational speed; (b) sun gear rotational speed $4200 \mathrm{r} / \mathrm{min}$.

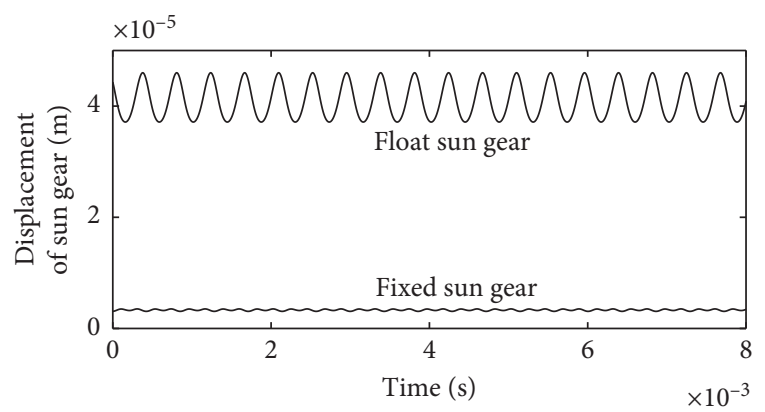

(a)

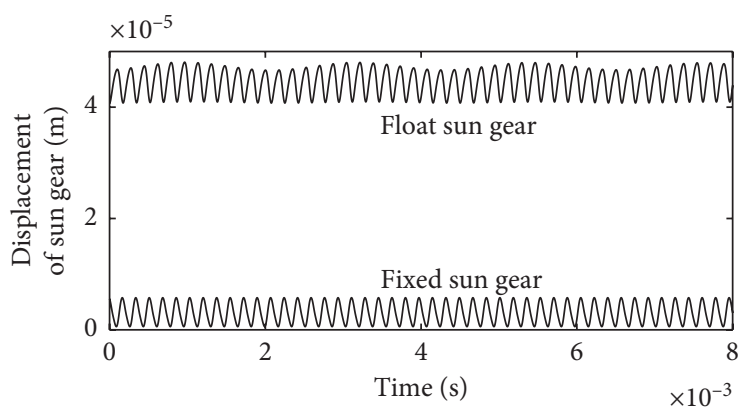

(b)

FIGURE 22: Comparisons of the translational displacement of sun gear. (a) $\omega_{m} \approx \omega_{r 1}$; (b) $\omega_{m} \approx \omega_{r 2}$.

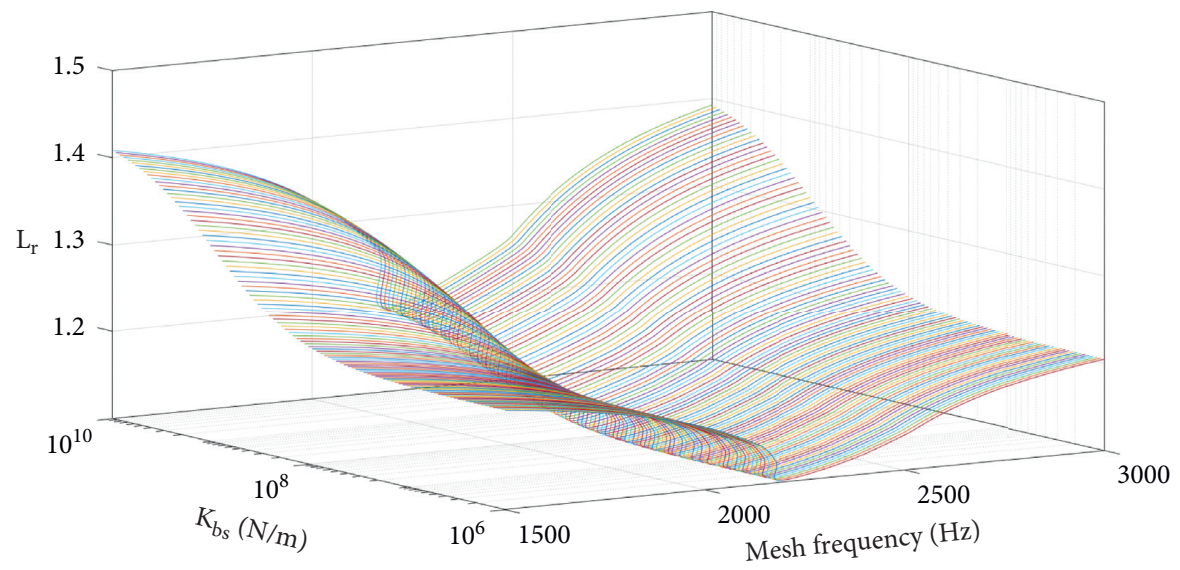

(a)

Figure 23: Continued. 


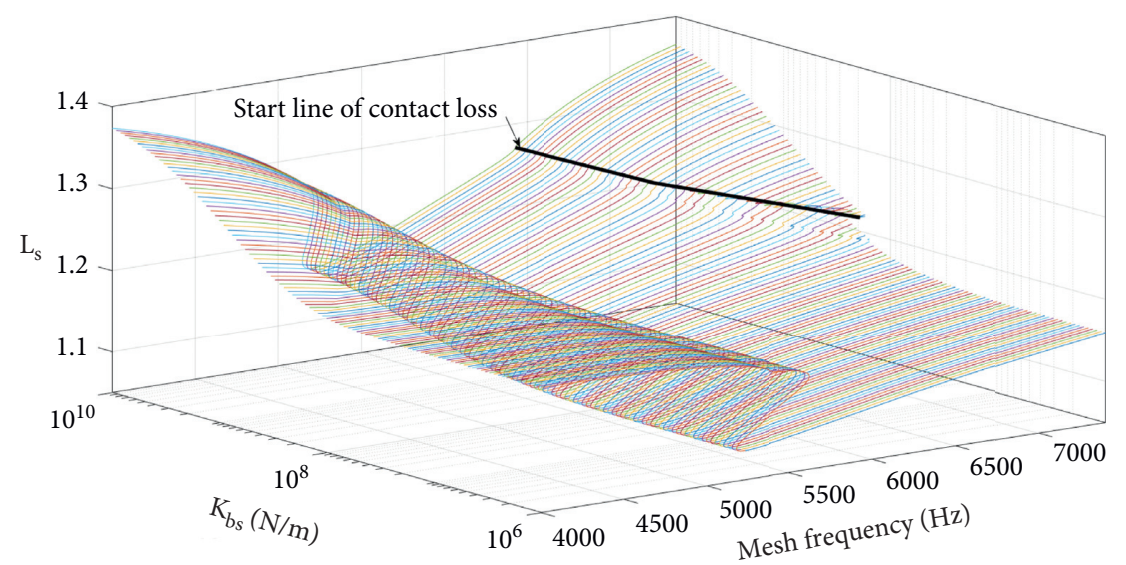

(b)

FIGURE 23: Load sharing factors for 5-planet system with varying support stiffness of sun gear. (a) $\omega_{m} \approx \omega_{r 1}$; (b) $\omega_{m} \approx \omega_{r 2}$.

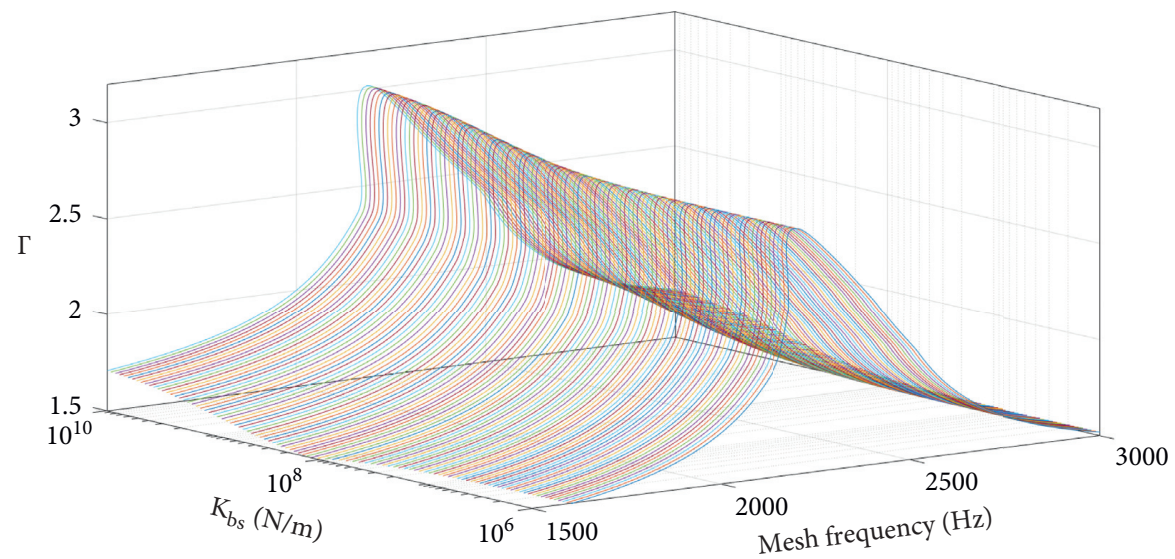

(a)

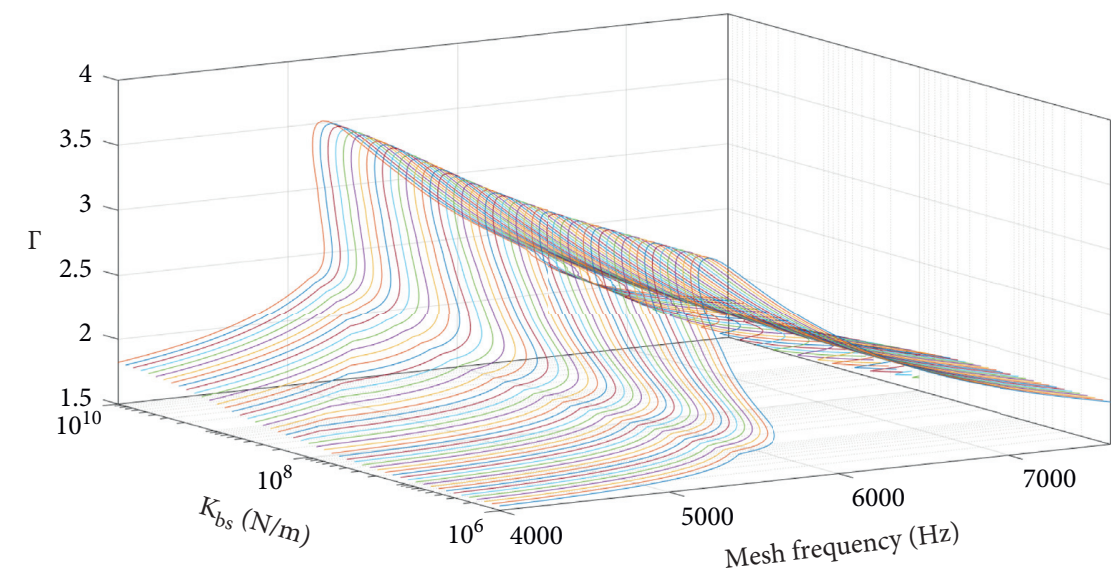

(b)

FIGURE 24: Dynamic load factors for 5-planet system with varying support stiffness of sun gear. (a) $\omega_{m} \approx \omega_{r 1}$; (b) $\omega_{m} \approx \omega_{r 2}$. 


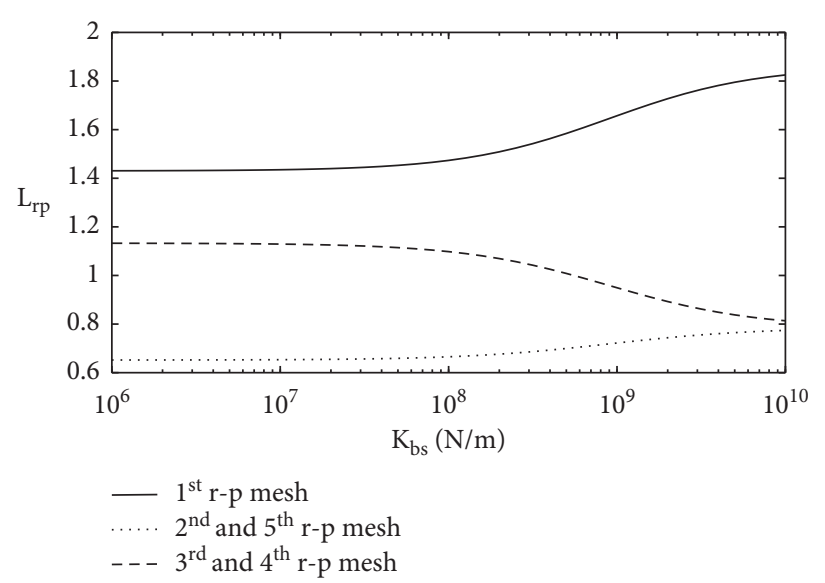

(a)

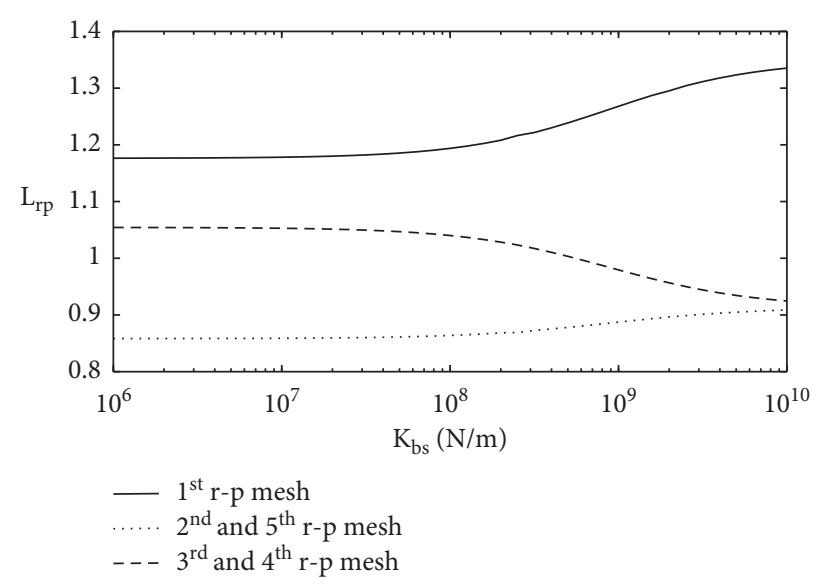

(b)

FIGURE 25: Effects of the support stiffness of the sun gear. (a) Quasistatic condition with low rotational speed; (b) sun gear rotational speed $4200 \mathrm{r} / \mathrm{min}$.

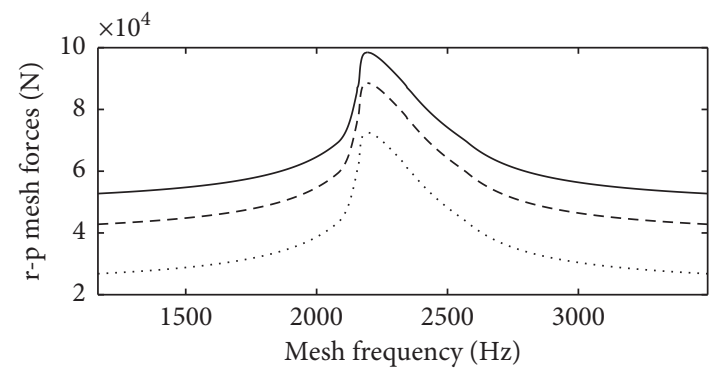

(a)

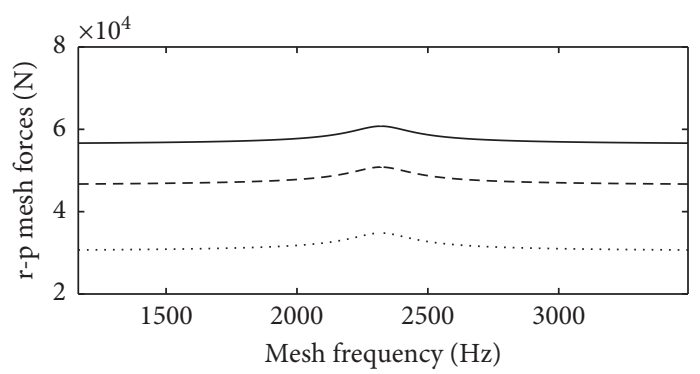

(c)

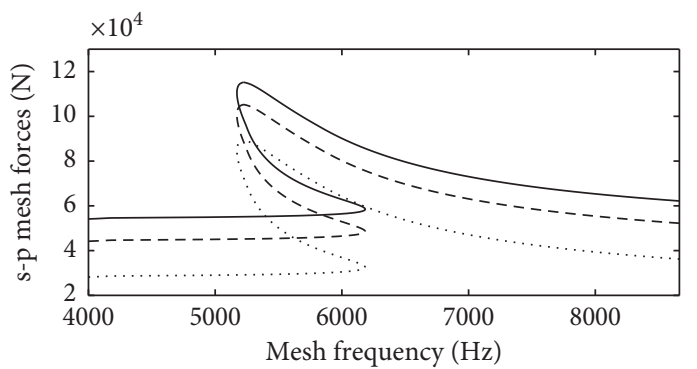

(b)

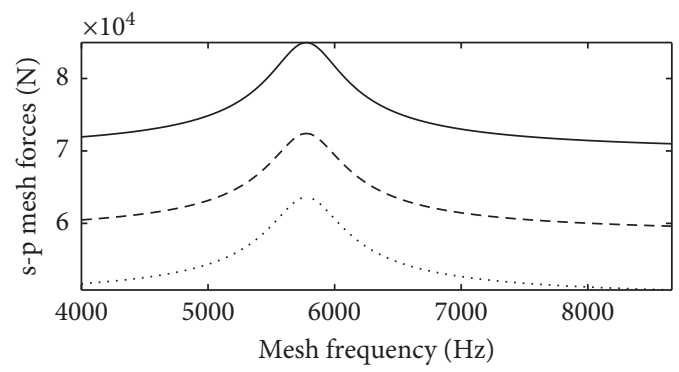

(d)

FIGURE 26: Comparisons of the amplitudes of mesh forces with and without TPMs. (-), the $1^{\text {st }}$ planet; (---), the $2^{\text {nd }}$ and the $5^{\text {th }}$ planets; (..), the $3 \mathrm{rd}$ and the $4^{\text {th }}$ planets, (a-b) without TPMs; (c-d) with TPMs.

4.6. Effects of Tooth Profile Modifications. TPM has been proved as an effective method to decrease the vibrations of PGT system [30-32]. Without TPM, tooth separations occur when $\omega_{m} \approx \omega_{r 1}, \omega_{r 2}$ and the "soften" phenomenon appears, as shown in Figures 26(a) and 26(b). Aiming at eliminating the tooth separations of both s-p and r-p meshes with $\omega_{m} \approx \omega_{r 1}, \omega_{r 2}$, simultaneously, the proper magnitudes of tooth profile modifications for the s-p and r-p meshes are $28 \mu \mathrm{m}$ and $15 \mu \mathrm{m}$, respectively. These optimal TMP amounts could be obtained by using the method presented in Ref. [31]. With proper TPM, the vibration amplitudes are dramatically decreased, and tooth separations are eliminated, as shown in Figures 26(c) and 26(d). Proper TPM suppresses the contributions of vibration to the mesh forces.

In Figures 27(a) and 27(b), the load sharing factors $L_{r}$ and $L_{s}$ are shown for the system with/without TPMs. By comparing the results obtained for the system without TPMs with the results obtained for the system with TPM, one can say TPM can help to eliminate the fluctuation of the load sharing factors with the change of mesh frequency, as shown in Figures 27(a) and 27(b). That means, with proper TPM, it is very reasonable to approximate the dynamical load sharing factors with the result obtained in quasistatic conditions. This conclusion provides the possibility to simplify 


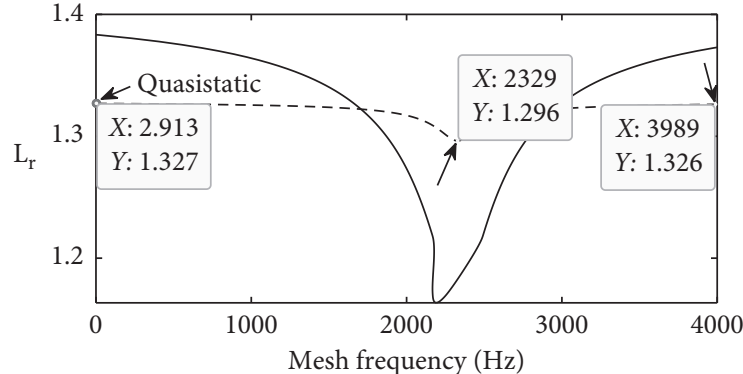

(a)

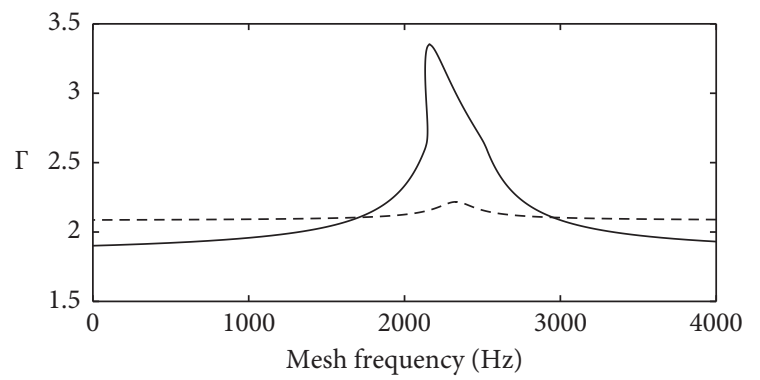

(c)

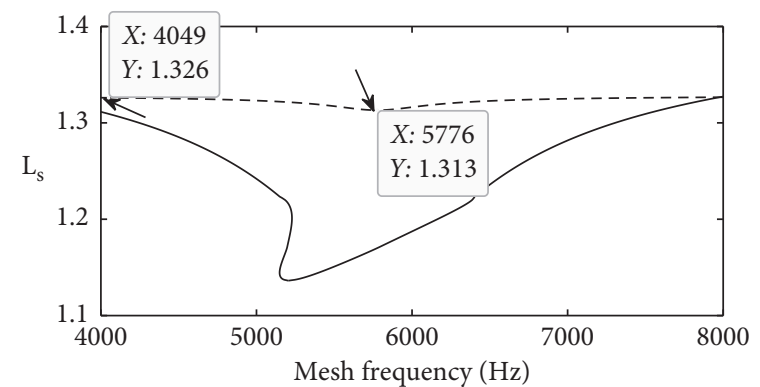

(b)

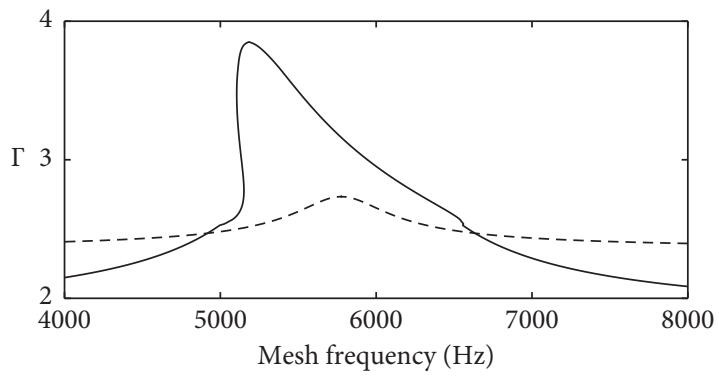

(d)

FIgURE 27: Comparisons of the load sharing factors and dynamic load factors of PGTs with and without TPMs. (-), without TPM; (---), with proper TPM. (a-b) Load sharing factors; (c-d) dynamic load factors.

the dynamic analysis to the static analysis on the dynamic load sharing problems.

Since the TPM is effective to decrease the system vibration amplitudes, TPM must be an effective method to decrease the dynamic load factors. As shown in Figures 27(c) and $27(\mathrm{~d})$, TPM dramatically decreases the dynamic load factors in the primary resonance frequency ranges.

The static and quasistatic results of the load sharing factors agree well with dynamic analysis, and the static and quasistatic analysis has advantages in analysis efficiency. However, dynamic analysis can offer a better understanding of the dynamic load factors. So, selection of static and dynamic analysis depends on the main focus on the PGTs.

\section{Conclusion}

In this study, a simplified discrete model is presented to investigate the load sharing among the planet meshes of PGTs with several type errors. Both of the cases of fixed and float sun gear are investigated to study the effects of the support stiffness of sun gear on the load sharing. Timevarying mesh stiffness and tooth separations are also considered. The method of multiple-scales (MMS) is used to obtain the response and closed-form expressions of mesh force are derived over the important mesh frequency ranges. From these expressions, the effects of several key factors such as the tooth thickness and pin position errors, applied torque, support stiffness of sun gear, and tooth profile modifications on dynamic load sharing behaviours are explored. The validation of MMS is obtained by the results of numerical integration and previously published predictions. Several conclusions are obtained:
(1) The amplitudes of dynamic mesh force are the function of vibration amplitude which is associated with the mesh frequency. That means the load sharing factors and the dynamic load factors are the functions of mesh frequency.

(2) For PGTs with different number of planet gears, the load sharing coefficients have similar trend versus mesh frequency. With equivalent tooth thickness error on the $1^{\text {st }}$ planet gear, the dynamic load sharing factor is proportional to the absolute magnitude of equivalent tooth thickness errors. While with the increasing of the planet gear number, the load sharing factors and dynamic load factors became more sensitive to the equivalent tooth thickness errors.

(3) With equivalent tooth thickness error on the $1^{\text {st }}$ planet gear, for 3-planet gear PGTs, the $2^{\text {nd }}$ and $3^{\text {rd }}$ planet gears carry equal load because of the geometric symmetry of position. For the same reason, the $2^{\text {nd }}$ and $4^{\text {th }}$ planet gears for 4-planet PGTs carry equal load. For 5- and 6-planet systems, the $2^{\text {nd }}$ and $N^{\text {th }}$ and $3^{\text {rd }}$ and $(N-1)^{\text {th }}$ planets carry equal load, respectively.

(4) Floating sun gear decreases the dynamic mesh load while optimizing the load sharing although the vibration amplitude of sun gear increases. That means floating sun gear is one of the effective methods to improve the dynamic load sharing with high priority.

(5) Large applied torque and low support stiffness of sun gear help to compensate the effects of manufacturing errors and to improve the load distribution. But large 
applied torque increases the nominal transmit mesh forces. Considering the static strength of gear tooth, increasing the applied torque to suppress the effects of equivalent tooth thickness error is not the first option.

(6) Tooth profile modification is effective to eliminate the tooth separation and decrease the vibration amplitudes. The amplitudes of mesh forces are also decreased by proper TPM. These effects further help to suppress the fluctuations of the dynamic load factor versus mesh frequency. With proper TPM, one can approximate the dynamical load sharing factors by the result obtained in quasistatic conditions.

\section{Abbreviations}

\section{C: $\quad$ Damping matrix}

$\mathbf{D}_{s p}, \mathbf{D}_{r p}$ : Dimensionless force vector caused by profile errors and TPM

$E_{c n}$ : Pin position error

$E_{p n}: \quad$ Tooth thickness error

$\mathbf{F}_{d}$ : $\quad$ Force vector caused by profile error

$\mathbf{F}_{m}: \quad$ Force vector caused by TPM

$\mathbf{F}_{t}$ : $\quad$ Force vector of applied torque

$J$ : $\quad$ Moments of inertia

$\mathbf{K}_{b}$ : $\quad$ Stiffness matrix of support

$\mathbf{K}_{m 0}$ : $\quad$ Mean mesh stiffness matrix

$\mathbf{K}_{m v}: \quad$ Variable mesh stiffness matrix

$\mathbf{K}_{s p}, \mathbf{K}_{r p}$ : Nondimensional mesh stiffness matrix

$L_{s p}, L_{r p}: \quad$ Load sharing coefficients

$L_{s}, L_{r}: \quad$ Load sharing factors

M: $\quad$ Mass matrix

$N$ : $\quad$ Number of planet gears

$T_{s}$ : Transmitted torque on sun gear

V: $\quad$ Modal matrix

Z: $\quad$ Tooth number of gears

$a_{i}: \quad$ Modal vibration amplitude

$\widehat{f}_{\text {esp }}, \hat{f}_{\text {erp }}:$ Additional mesh forces induced by errors

$\hat{f}_{m s p}, \hat{f}_{m r p}$ : Additional mesh forces induced by TPM

$f_{\delta s p}, f_{\delta r p}$ : Peak values of mesh forces during a mesh period

$e_{s p}, e_{r p}: \quad$ Equivalent tooth thickness errors

$h_{s p}, h_{r p}: \quad$ TPM functions

$i: \quad$ DOFs index

$k_{b s}: \quad$ Support stiffness of sun gear

$k_{s p}, k_{r p}: \quad$ Mesh stiffness

$k_{n u}$ : Torsional stiffness

$m: \quad$ mass

$p: \quad$ Planet gear index

$r_{n}: \quad$ Base radii

$t: \quad$ Time

$u_{n}$ : $\quad$ Deflection of the gear bodies along the line of action

$x_{s}, y_{s}: \quad$ Translational motions of sun gear

$\Omega: \quad$ Rotate speed

$\Gamma$ : $\quad$ Dynamic load factors

$\Theta: \quad$ Tooth separation function

$\Psi_{p}$ : $\quad$ Position angle of planet gear

$\begin{array}{ll}\alpha_{s}, \alpha_{r}: & \text { Pressure angles } \\ \varphi: & \text { Angle between pin position errors and the mesh } \\ \Upsilon: & \text { line } \\ \omega_{m}: & \text { Initial phase of errors } \\ \omega_{i}: & \text { Mesh frequency } \\ \delta: & \text { Natural frequency } \\ \zeta: & \text { Mesh deflection } \\ & \text { Damping ratio. }\end{array}$

\section{Data Availability}

The MATLAB data used to support the findings of this study are available from the corresponding author upon request.

\section{Conflicts of Interest}

The authors declare that they have no conflicts of interest.

\section{Acknowledgments}

The authors gratefully acknowledge the support by the Scientific Research Fund of High-Level Talents in Nanjing Institute of Technology (YKJ 201951).

\section{References}

[1] A. Kahraman and S. Vijayakar, "Effect of internal gear flexibility on the quasi-static behavior of a planetary gear set," Journal of Mechanical Design, vol. 123, no. 3, p. 408, 2001.

[2] J. Margielewicz, D. Gąska, and G. Litak, "Modelling of the gear backlash," Nonlinear Dynamics, vol. 97, no. 1, pp. 355-368, 2019.

[3] W. Luo, B. Qiao, Z. Shen et al., "Time-varying mesh stiffness calculation of a planetary gear set with the spalling defect under sliding friction," Meccanica, vol. 55, no. 1, pp. 245-260, 2020.

[4] W. Luo, B. Qiao, Z. Shen, Z. Yang, H. Cao, and X. Chen, "Investigation on the influence of spalling defects on the dynamic performance of planetary gear sets with sliding friction," Tribology International, vol. 154, Article ID 106639, 2021.

[5] C. G. Cooley and R. G. Parker, "A review of planetary and epicyclic gear Dynamics and vibrations Research," Applied Mechanics Reviews, vol. 66, no. 4, 2014.

[6] A. Bodas and A. Kahraman, "Influence of carrier and gear manufacturing errors on the static load sharing behavior of planetary gear sets," JSME International Journal Series C, vol. 47, no. 3, p. 908, 2004.

[7] A. Singh, "Application of a system level model to study the planetary load sharing behavior," Journal of Mechanical Design, vol. 127, no. 3, p. 469, 2005.

[8] A. N. Montestruc, "Influence of planet pin stiffness on load sharing in planetary gear drives," Journal of Mechanical Design, vol. 133, no. 1, 2011.

[9] C. Gill-Jeong and R. G. Parker, "Influence of bearing stiffness on the static properties of a planetary gear system with manufacturing errors," KSME International Journal, vol. 18, no. 11, p. 1978, 2004.

[10] H. Ligata, A. Kahraman, and A. Singh, "An experimental study of the influence of manufacturing errors on the planetary gear stresses and planet load sharing," Journal of $\mathrm{Me}$ chanical Design, vol. 130, no. 4, 2008. 
[11] A. Singh, A. Kahraman, and H. Ligata, "Internal gear strains and load sharing in planetary transmissions: model and experiments," Journal of Mechanical Design, vol. 130, no. 7, 2008.

[12] J.-G. Kim, Y.-J. Park, G.-H. Lee, and J.-H. Kim, “An experimental study on the effect of carrier pinhole position errors on planet gear load sharing," International Journal of Precision Engineering and Manufacturing, vol. 17, no. 10, p. 1305, 2016.

[13] H. Ligata, A. Kahraman, and A. Singh, "A closed-form planet load sharing formulation for planetary gear sets using a translational analogy," Journal of Mechanical Design, vol. 131, no. 2, 2009.

[14] A. Singh, "Load sharing behavior in epicyclic gears: physical explanation and generalized formulation," Mechanism and Machine Theory, vol. 45, no. 3, p. 511, 2010.

[15] A. Singh, "Epicyclic load sharing map-development and validation," Mechanism and Machine Theory, vol. 46, no. 5, p. 632, 2011.

[16] A. Kahraman, "Load sharing characteristics of planetary transmissions," Mechanism and Machine Theory, vol. 29, 1994.

[17] X. Y. Gu and P. Velex, "A lumped parameter model to analyse the Dynamic load sharing in planetary gears with planet errors," Applied Mechanics and Materials, vol. 86, pp. 374379, 2011.

[18] X. Gu and P. Velex, "A dynamic model to study the influence of planet position errors in planetary gears," Journal of Sound and Vibration, vol. 331, no. 20, p. 4554, 2012.

[19] X. Gu and P. Velex, "On the dynamic simulation of eccentricity errors in planetary gears," Mechanism and Machine Theory, vol. 61, p. 14, 2013.

[20] C. Yuksel and A. Kahraman, "Dynamic tooth loads of planetary gear sets having tooth profile wear," Mechanism and Machine Theory, vol. 39, no. 7, p. 695, 2004.

[21] F. Chaari, T. Fakhfakh, R. Hbaieb et al., "Influence of manufacturing errors on the dynamic behavior of planetary gears," International Journal of Advanced Manufacturing Technology, vol. 27, 2006.

[22] M. Iglesias, A. Fernández, A. De-Juan, R. Sancibrián, and P. García, "Planet position errors in planetary transmission: effect on load sharing and transmission error," Frontiers of Mechanical Engineering, vol. 8, no. 1, p. 80, 2013.

[23] G.-J. Cheon and R. G. Parker, "Influence of manufacturing errors on the dynamic characteristics of planetary gear systems," KSME International Journal, vol. 18, no. 4, p. 606, 2004.

[24] M. Shuai, T. Zhang, and J. Guo, "Analytical investigation on load sharing characteristics of herringbone planetary gear train with flexible support and floating sun gear," Mechanism and Machine Theory, vol. 144, 2020.

[25] S. Mo, Z. Yue, Z. Feng, L. Shi, Z. Zou, and H. Dang, "Analytical investigation on load-sharing characteristics for multipower face gear split flow system," Proceedings of the Institution of Mechanical Engineers, Part C: Journal of Mechanical Engineering Science, vol. 234, no. 2, p. 676, 2020.

[26] Y.-J. Park, J.-G. Kim, G.-H. Lee, and S. B. Shim, "Load sharing and distributed on the gear flank of wind turbine planetary gearbox," Journal of Mechanical Science and Technology, vol. 29, no. 1, p. 309, 2015.

[27] F. Ren, D. Qin, T. C. Lim, and S. Lyu, "Study on dynamic characteristics and load sharing of a herringbone planetary gear with manufacturing errors," International Journal of Precision Engineering and Manufacturing, vol. 15, no. 9, p. $1925,2014$.
[28] F. Ren, G. Luo, G. Shi, X. Wu, and N. Wang, "Influence of manufacturing errors on dynamic floating characteristics for herringbone planetary gears," Nonlinear Dynamics, vol. 93, no. 2, p. 361, 2018.

[29] C.-J. Bahk and R. G. Parker, "Analytical solution for the nonlinear Dynamics of planetary gears," Journal of Computational and Nonlinear Dynamics, vol. 6, no. 2, 2010.

[30] C. J. Bahk and R. G. Parker, "Analytical investigation of tooth profile modification effects on planetary gear dynamics," Mechanism and Machine Theory, vol. 70, 2013.

[31] C. Xun, X. Long, and H. Hua, "The Effects of multi-mesh tooth profile modifications on planetary gear vibration," in Proceedings of the ASME International Mechanical Engineering Congress and Exposition, Phoenix, AZ, USA, 2016.

[32] C. Xun, X. Long, and H. Hua, "Effects of random tooth profile errors on the dynamic behaviors of planetary gears," Journal of Sound and Vibration, vol. 415, p. 91, 2018.

[33] H. Dai, X. Long, F. Chen, and C. Xun, "An improved analytical model for gear mesh stiffness calculation," Mechanism and Machine Theory, vol. 159, no. 1, Article ID 104262, 2021.

[34] A. Kahraman, "Natural modes of planetary gear trains," Journal of Sound and Vibration, vol. 173, no. 1, p. 125, 1994.

[35] H. N. Ali and M. Deant, Nonlinear Oscillations, Wiley, Hoboken, NJ, USA, 1979. 